\title{
Microbial sensing by goblet cells controls immune surveillance of luminal antigens in the colon
}

\author{
KA Knoop ${ }^{1}$, KG McDonald ${ }^{1}, \mathrm{~S} \mathrm{McCrate}^{1}, \mathrm{JR}_{\mathrm{McDole}}{ }^{2}$ and RD Newberry ${ }^{1}$
}

The delivery of luminal substances across the intestinal epithelium to the immune system is a critical event in immune surveillance, resulting in tolerance to dietary antigens and immunity to pathogens. How this process is regulated is largely unknown. Recently goblet cell-associated antigen passages (GAPs) were identified as a pathway delivering luminal antigens to underlying lamina propria (LP) dendritic cells in the steady state. Here, we demonstrate that goblet cells (GCs) form GAPs in response to acetylcholine (ACh) acting on muscarinic ACh receptor 4. GAP formation in the small intestine was regulated at the level of ACh production, as GCs rapidly formed GAPs in response to ACh analogs. In contrast, colonic GAP formation was regulated at the level of GC responsiveness to ACh. Myd88-dependent microbial sensing by colonic GCs inhibited the ability of colonic GCs to respond to Ach to form GAPs and deliver luminal antigens to colonic LP-antigen-presenting cells (APCs). Disruption of GC microbial sensing in the setting of an intact gut microbiota opened colonic GAPs, and resulted in recruitment of neutrophils and APCs and production of inflammatory cytokines. Thus GC intrinsic sensing of the microbiota has a critical role regulating the exposure of the colonic immune system to luminal substances.

\section{INTRODUCTION}

The non-follicle-bearing epithelium lining the gastrointestinal tract is the largest environmentally exposed surface in the body. This epithelium functions as a site for nutrient absorption, as a barrier separating the underlying immune system from potential pathogens, and as a site for immune surveillance of substances derived from the lumen. ${ }^{1,2}$ How immune surveillance at the non-follicle-bearing epithelium is regulated to allow education of the immune system to the luminal contents while preventing unwanted or unnecessary exposures is poorly understood.

Goblet cells (GCs) are specialized epithelial cells found in the non-follicle-bearing epithelium of the gastrointestinal tract. GCs have an established role in innate immunity by secreting mucins, a major component of the mucous layer, which provides a first line of defense against physical and chemical injury, and protects against the invasion by pathogens. ${ }^{3,4}$ GCs produce and secrete other biologically active products contributing to innate immunity such as trefoil peptides, resistinlike molecule $\beta$, and the $\mathrm{Fc}-\gamma$-binding protein, which respectively function to promote epithelial restitution, inhibit intestinal nematode chemotaxis, and stabilize the mucous layer. ${ }^{5-7}$ Recently, it was discovered that when small intestine (SI) GCs secrete, they form goblet cell-associated antigen passages (GAPs) and deliver small soluble luminal antigens to lamina propria (LP) dendritic cells (DCs). ${ }^{8}$ GAPs were found to be the dominant mechanism for delivering luminal antigen to SI LP-DCs in the healthy steady state, as luminal antigen delivery to SI LP-DCs was undetectable when GCs and GAPs were absent. ${ }^{8}$ Furthermore SI GAPs were dynamic, appearing and disappearing during in vivo imaging, and inducing GC secretion augmented GAPs and antigen delivery to SI LP-DCs, indicating that this antigen delivery mechanism may be manipulated and regulated. Notably, GAPs were not seen in the colon of multiple strains of specific pathogen-free (SPF)housed mice despite the presence of abundant GCs and mucus indicative of GC secretion. ${ }^{8}$ These observations suggest that there may be intrinsic differences between SI and colonic GCs, and/or that environmental differences between the SI and colon may influence the ability of GCs to form GAPs. In addition, the absence of colonic GAPs in the setting of abundant mucus could suggest that not all GC secretion is linked to GAP

${ }^{1}$ Department of Internal Medicine, Washington University School of Medicine, St Louis, Missouri, USA and ${ }^{2}$ Department of Pathology and Immunology, Washington University School of Medicine, St Louis, Missouri, USA. Correspondence: RD Newberry (rnewberry@im.wustl.edu)

Received 11 April 2014; accepted 3 June 2014; published online 9 July 2014. doi:10.1038/mi.2014.58 
formation and, by extension, linked with antigen delivery. This suggests that GCs could secrete mucus to maintain the barrier and not expose the immune system to luminal contents when conditions are unfavorable for antigen delivery.

Using the above observations as a background, we investigated how GAP formation and antigen delivery to the LP immune system is regulated, including the stimuli for inducing GCs to form GAPs in the basal state. Here, we demonstrate that GAP formation is driven by acetylcholine (ACh) acting on the muscarinic ACh receptor (mAChR) 4. Colonic GC sensitivity to $\mathrm{mAChR}_{4}$ signals, GAP formation, and luminal antigen delivery was rapidly suppressed by GC intrinsic sensing of the luminal microbiota, demonstrating a rapid and local control of antigen delivery in response to the luminal environment. Overriding GC microbial sensing to open colonic GAPs resulted in the influx of leukocytes and the production of inflammatory cytokines in the setting of the normal, nonpathogenic, microbiota. These findings identify a central role for GCs controlling the exposure of the immune system to luminal antigens and guiding immune surveillance at the nonfollicle-bearing epithelium.

\section{RESULTS}

GAP formation is driven by $\mathrm{mAChR}_{4}$ signaling in the $\mathrm{SI}$ in the basal state and in the colon following treatment with antibiotics

GAP formation was observed to be linked with GC secretion induced by the pan-mAChR, pan-nicotinic ACh receptor agonist carbamylcholine (CCh). ${ }^{8}$ Employing the in vivo imaging approach used to identify GAPs, ${ }^{8}$ we evaluated the stimuli inducing GAPs in the SI in the basal state. Following luminal administration of fluorescent $10 \mathrm{kDa}$ dextran, GAPs were readily observed in the SI of SPF-housed C57BL/6 mice and were significantly augmented following treatment with CCh (Figure 1a,b). GAPs were not augmented by luminal (Figure 1b) or systemic (not shown) administration of cholera toxin, a well-studied GC secretagogue, ${ }^{9}$ demonstrating GAP formation is not linked with stimulation by all GC secretagogues. SI GAPs were effectively inhibited by atropine, a pan$\mathrm{mAChR}$ antagonist (Figure 1a,b). Cholingeric agonists are known to induce GC secretion by acting directly on GCs, ${ }^{10}$ suggesting that GAP formation could be driven by ACh acting directly on mAChRs expressed by GCs. Five mAChR subtypes, $\mathrm{mAChR}_{1-5}$, are expressed in mammals. ${ }^{11}$ Gene expression microarrays performed on flow cytometrically sorted GCs from the SI and colon revealed that intestinal GCs express $\mathrm{mAChR}_{3}$ and $\mathrm{mAChR}_{4}$ (Supplementary Figure S1 online), which was confirmed by using quantitative real-time PCR (Figure 1c). Selective pharmacologic blockade of $\mathrm{mAChR}_{4}$ by administration of tropicamide (Figure 1a,b) or himbacine (not shown), but not blockade of $\mathrm{mAChR}_{3}$ by piperidine (Figure 1a,b) or 4-DAMP (not shown), effectively inhibited GAP formation in the SI, demonstrating that in the basal state ACh signaling via $\mathrm{mAChR}_{4}$ drives GAP formation in the SI.

GAPs were found in the SI, but not in the colon, of multiple conventionally housed mouse strains. ${ }^{8}$ The abundance of the microbiota is a major difference in the luminal environment between the SI and colon, with the SI harboring $\sim 10^{3}$ bacteria and the colon harboring $\sim 10^{11}$ bacteria per gram of intestinal contents. ${ }^{12}$ The microbiota contributes to multiple processes including epithelial growth, metabolism, and immune stimulation, ${ }^{12}$ any of which could have a role in modulating GC secretion and GAP formation. To evaluate a role for the microbiota in GAP regulation, we treated mice with oral antibiotics to disrupt the enteric flora. We observed that SI GAPs were unchanged, but trans-epithelial columns of dextran became apparent in the colon (Figure 1d). The columns of dextran appeared on the surface epithelium and throughout the colonic crypts, were approximately the size of an epithelial cell, and contained a diamidino-2-phenylindole-positive nuclei (Supplementary Movie S1). These columns were absent in the colon of antibiotic-treated mice in which mouse atonal homolog 1 (Math1), a transcription factor required for GC development, ${ }^{13}$ was inducibly deleted in epithelial cells resulting in the depletion of GCs, or Math $1^{\text {i } \Delta \text { vil }}$ mice (Figure 1e). In the steady state, the colonic epithelium does not contain Paneth cells, and within the colonic epithelium Math 1 is almost exclusively expressed by GCs. ${ }^{14}$ These columns colocalized with colonic epithelial cells with GC morphology expressing red fluorescent protein in $\mathrm{dtom}^{\mathrm{i} \text { Mathl }}$ reporter mice, which labels Math1-expressing cells with the tdTomato red fluorescent protein following injection with RU486 (Figure 1f). Therefore, disruption of the enteric microbiota by antibiotics allowed colonic GCs to acquire the luminal fluorescent model antigen and form GAPs.

Unlike the SI, colonic GCs in conventionally housed mice were relatively unresponsive to cholinergic agonists and did not form GAPs at doses of $\mathrm{CCh}$ that could be given in vivo (Figure 1g,i). However, similar to SI GCs from conventionally housed mice, colonic GCs from antibiotic-treated mice formed GAPs in the basal state, and were sensitive to exogenous cholinergic agonists in vivo to induce further GAP formation (Figure 1h,i). Furthermore, colonic GAPs in antibiotic-treated mice were inhibited by $\mathrm{mAChR}_{4}$, but not $\mathrm{mAChR}_{3}$, blockade (Figure 1h,i), indicating that similar pathways were inducing GAPs in the SI in the basal state and in the colon following antibiotic treatment, and suggesting that antibiotic treatment relieved the suppression of colonic GC responsiveness to $\mathrm{mAChR}_{4}$ signals.

\section{GC intrinsic Myd88-dependent microbial sensing inhibits GAP formation in the colon}

To assess whether host sensing of the luminal microbiota, as opposed to other properties of the microbiota or other properties of antibiotic treatment, was regulating the ability of GCs to respond to ACh and form GAPs, we evaluated the colon of germ-free (GF) mice, which lack a luminal microbiota without antibiotic treatment, and Myd $88^{-1-}$ mice, which have impaired responses to microbe-associated molecular pattern motifs via the toll-like receptors (TLRs). We observed that, like the colon of antibiotic-treated mice, colonic GAPs formed in GF mice and Myd88 ${ }^{-1-}$ mice, and that the colonic GAPs in 
a
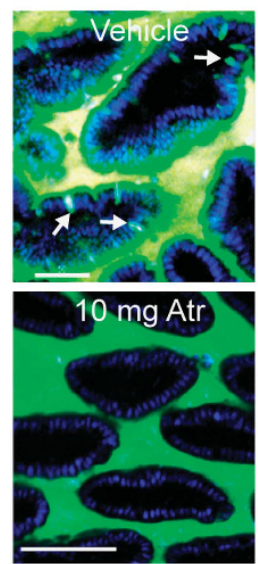

d

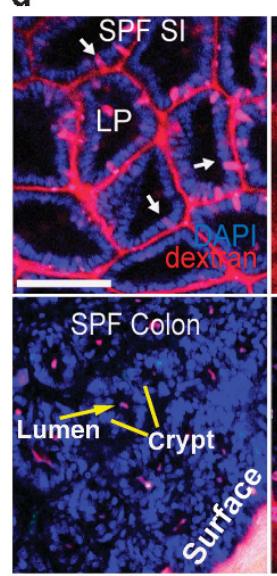

h
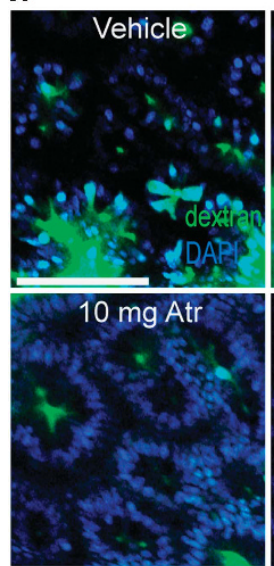

SPF SI
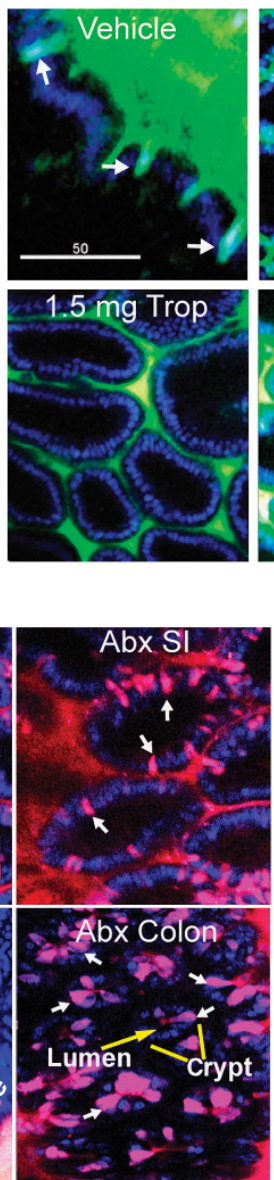

Abx colon
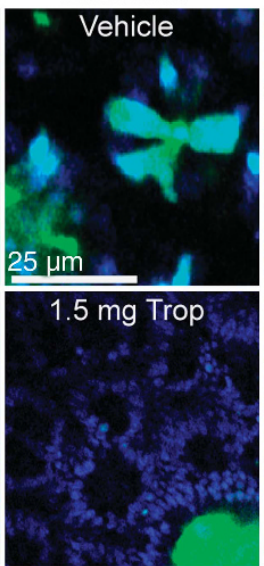
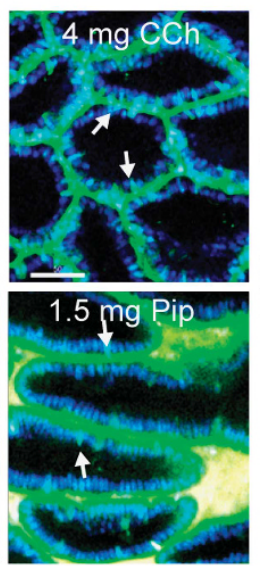

e
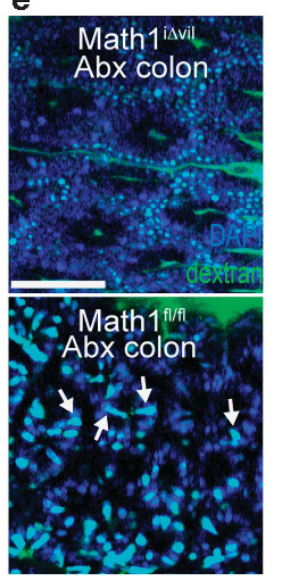

b

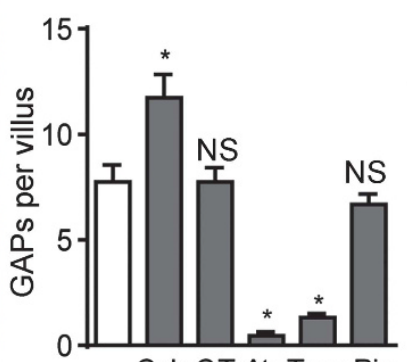

- Cch CT Atr Trop Pip

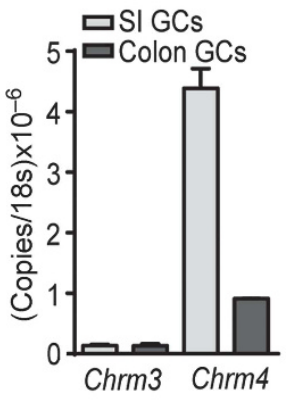

g SPF Colon

f
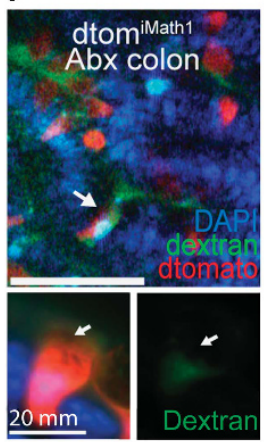

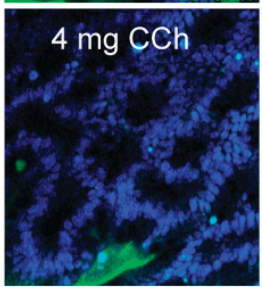

i
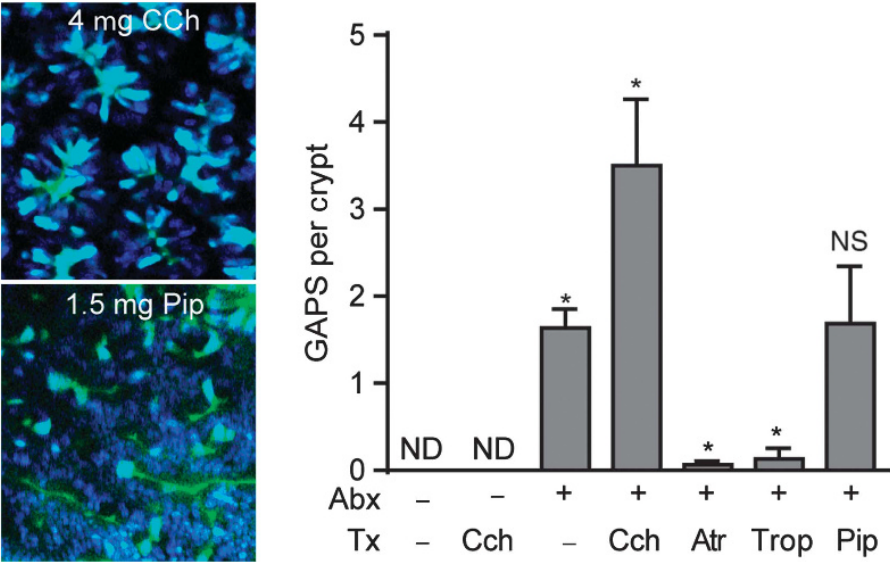

Figure 1 Goblet cell-associated antigen passage (GAP) formation is driven by muscarinic acetylcholine receptor $4\left(\mathrm{mAChR}_{4}\right)$ signaling in the small intestine (SI) at the basal state and in the colon following treatment with antibiotics. (a) Intravital two-photon (2P) images of the SI following the luminal administration of dextran (green) and diamidino-2-phenylindole (DAPI) (blue) and (b) number of GAPs per villus from mice treated with carbamylcholine

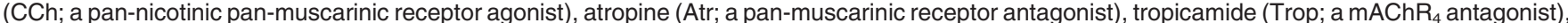
piperidine (Pip; a mAChR ${ }_{3}$ antagonist), or cholera toxin (CT; a goblet cell (GC) secretagogue, in $\mathbf{b}$ only). (c) Quantitative real-time RNA for the genes encoding $\mathrm{mAChR}_{3}$ and $\mathrm{mAChR}_{4}$ (Chrm3 and Chrm4, respectively) from fluorescence-activated cell-sorted SI and colonic GCs. (d) $2 \mathrm{P}$ images of the SI and colon following the administration of luminal dextran (red) and DAPI (blue) in specific pathogen-free (SPF)-housed mice untreated or treated with antibiotics (Abx). (e) 2P images of the colon of Math $1^{\text {i vvil }}$ mice or littermate controls treated with tamoxifen and antibiotics following the administration of luminal dextran (green) and DAPI (blue). (f) $2 \mathrm{P}$ and fluorescent immunohistochemistry images of the colon following the administration of luminal dextran (green) and DAPI (blue) in dtom iMath1 mice treated with RU486 and antibiotics demonstrating dextran (green) colocalizing with Math1-expressing epithelial cells with GC morphology (red). ( $(\mathbf{g}$ and $\mathbf{h}$ ) 2P images of the colon and (i) number of GAPs per crypt from untreated SPF-housed mice and antibiotic-treated SPF-housed mice given vehicle, cholinergic agonists, or non-specific or specific mAChR antagonists. White arrows denote GAPs, yellow arrows denote the colonic crypt lumen. Data are presented as the mean \pm s.e.m., ${ }^{\star} P<0.05$. LP, lamina propria; ND, not detected; NS, not significant. $n=3$ or more mice for each condition in panels a-d, and $\mathbf{g}-\mathbf{i}, n=2$ in panels $\mathbf{e}$ and $\mathbf{f}$. Scale bar $=50 \mu \mathrm{m}$, unless otherwise noted. 

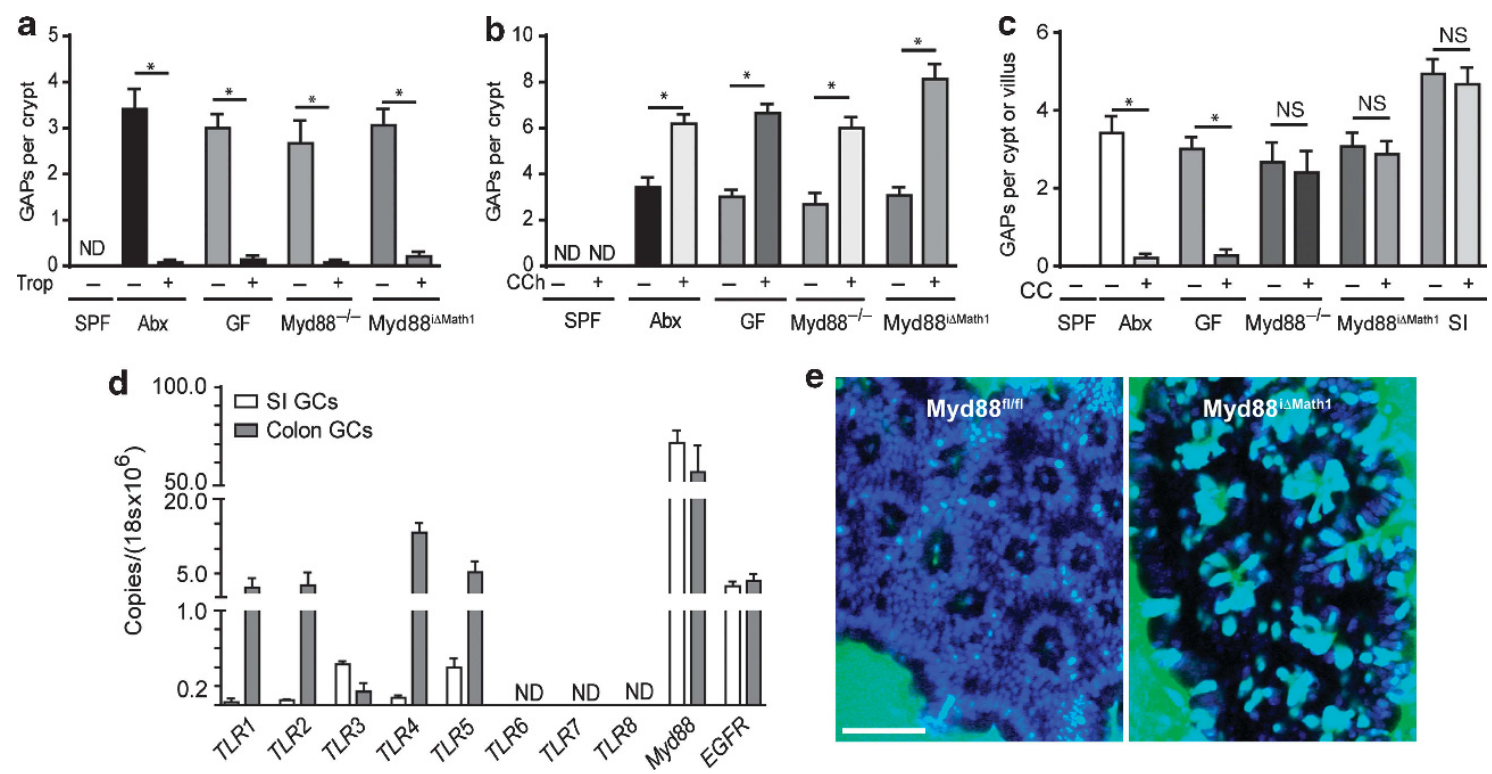

Figure 2 Goblet cell (GC) intrinsic Myd88-dependent microbial sensing inhibits goblet cell-associated antigen passages (GAPs) in the colon, but not the small intestine (SI). (a-c) Number of GAPs per colonic crypt or SI villus from specific pathogen-free (SPF)-housed mice, mice treated with antibiotics

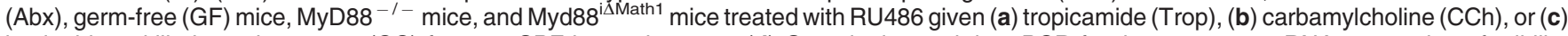
luminal heat-killed cecal contents (CC) from an SPF-housed mouse. (d) Quantitative real-time PCR for the messenger RNA expression of toll-like receptors (TLRs), Myd88, and the epidermal growth factor receptor (EGFR) from fluorescence-activated cell-sorted SI or colonic goblet cells isolated from SPF-housed mice. (e) Two-photon images from Myd88 $8^{i \Delta a t h 1}$ mice or Myd88 $8^{\mathrm{fl} / \mathrm{fl}}$ littermates treated with RU486. Data are presented as the mean \pm s.e.m., ${ }^{*} P<0.05$. ND, not detected; NS, not significant. $n=4$ or more mice for each condition in panels a-d, panel $\mathbf{e}$ is representative of one of three mice from each condition. Scale bar $=50 \mu \mathrm{m}$.

these mice were inhibited by $\mathrm{mAChR}_{4}$ antagonists (Figure 2a). Although colonic GCs were slightly increased in GF and Myd $88^{-I-}$ mice, this increase is insufficient to explain the marked appearance of colonic GAPs in these mice; in addition, GCs were not increased in antibiotic-treated mice, which had a similar marked increase in GAPs (Supplementary Figure S2a). Similar to the SI of SPF-housed mice, the exogenous cholinergic agonist CCh induced an increase in colonic GAPs in GF and SPF-housed Myd88 $8^{-1-}$ mice, indicating that colonic GCs in mice with a reduced microbial load or altered microbial sensing became responsive to $\mathrm{ACh}$ via $\mathrm{mAChR}_{4}$ (Figure $2 \mathbf{b}$ ). Muscarinic AChR expression was unchanged in Myd88 ${ }^{-1-}$ and antibiotic-treated mice (Supplementary Figure S1d), and, consistent with this unresponsiveness being mediated by a rapid blockade of the $\mathrm{mAChR}_{4}$ signaling pathway due to microbial sensing, we observed that heat-killed cecal contents (CC) isolated from SPF-housed mice inhibited colonic GAP formation within minutes upon intraluminal administration in GF mice and antibiotic treated mice, but not in $\mathrm{Myd} 88^{-1-}$ mice (Figure 2c). Real-time PCR analysis of the CCs revealed no significant differences in the amount of bacteria present or changes in the ratios of the major phyla between SPF-housed wild-type mice and Myd $88^{-1-}$ mice (Supplementary Figure S2b,c), suggesting the alteration in microbial sensing in Myd88 $8^{-1-}$ mice, not differences in the microbiota account for the formation of colonic GAPs. Gene expression microarrays (Supplementary Figure S1c) and quantitative real-time PCR (Figure 2d) performed on RNA isolated from GCs revealed that
GCs express TLRs 1-5 and Myd88, with colonic GCs expressing much higher levels of TLRs 1, 2, 4, and 5, and SI GCs expressing slightly higher levels of TLR3. Consistent with GC intrinsic microbial sensing regulating GAP formation, acute exposure to ligands for TLRs expressed by GCs inhibited colonic GAP formation in GF mice (Supplementary Figure S2d,e). We also observed that heat-killed CC or lipopolysaccharides (LPS) could rapidly inhibit secretion in response to cholinergic stimuli in the LS174T GC line (Supplementary Figure S3a), implicating that GC intrinsic microbial sensing was able to acutely inhibit $\mathrm{mAChR}$ responses. To explore the role of GC intrinsic microbial sensing in regulating colonic GAP formation in vivo, we generated mice with an inducible deletion of Myd88 in Math1expressing cells, which is largely restricted to GCs in the colon, or Myd8 $8^{\text {iSMath1 } 1}$ mice. Upon treatment with RU846, to delete Myd88 in GCs, these mice spontaneously formed GAPs in the colon (Figure 2e). Furthermore, colonic GAPs in Myd88 $8^{\text {iAMath1 }}$ mice could be augmented by treatment with $\mathrm{CCh}$, a cholinergic agonist, and inhibited by $\mathrm{mAChR}_{4}$ antagonists (Figure 2a,b), but were not inhibited by treatment with CC (Figure 2c), consistent with GC intrinsic Myd88-dependent sensing inhibiting GCs ability to respond to $\mathrm{mAChR}_{4}$ signals. Heatkilled CC from SPF-housed mice did not inhibit SI GAP formation (Figure 2c), and consistent with this, we did not see

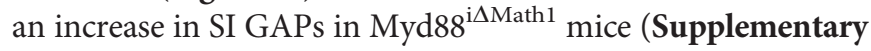
Figure S4), indicating that GC intrinsic sensing of the microbiota has a less prominent role in inhibiting SI GAPs in the steady state. 
GC intrinsic microbial sensing activates the epidermal growth factor receptor (EGFR), p42/p44 mitogen-activated protein kinase (MAPK), and suppresses $\mathrm{mAChR}_{4}$ dependent GAP formation

In epithelial cells, ligation of TLRs, the pattern recognition alters upstream of Myd88, can activate the EGFR ${ }^{15}$ and p42/p44 MAPK. ${ }^{16}$ In lacrimal gland GCs, p42/p44 MAPK activation alters mAChR-induced secretion, ${ }^{17-19}$ suggesting that p42/p44 MAPK activation, through the EGFR, is a potential link between Myd88-dependent microbial sensing and inhibition of mAChR-induced GAP formation. We observed that GCs express messenger RNAs for the EGFR (Figure 2d; Supplementary Figure S1c), and consistent with this pathway blocking secretion and GAP formation, EGF activated the EGFR and p42/p44 MAPK and inhibited cholinergic agonistinduced secretion in a cell-intrinsic manner in the LS174T GC line (Supplementary Figure S3). Furthermore, microbial products suppressed mucus secretion in a cell-intrinsic manner, and this correlated with increased levels of phosphorylated EGFR and p42/p44 MAPK in the LS174T GC line (Supplementary Figure S3).

Activation of the EGFR and p42/p44 MAPK was increased in vivo in colonic GCs and colonic epithelium of mice with an intact microbiota and intact microbial sensing (Figure 3a,b). Further supporting GC intrinsic microbial sensing activating the EGFR and p42/p44 MAPK, deletion of Myd88 in GCs resulted in decreased activation of EGFR and p42/p44 MAPK in GCs in vivo in conventionally housed mice (Figure 3c). Inhibition of EGFR activation (EGFRi) or p42/p44 MAPK activation (MAPKi) reversed the suppression of GC responses to ACh imposed by host microbial sensing, and resulted in the formation of colonic GAPs in an $\mathrm{mAChR}_{4}$-dependent manner in conventionally housed mice (Figure 3d; Supplementary Movie 2). Furthermore, EGF could supplant the signals delivered by microbial sensing and rapidly inhibit colonic GAPs in vivo in antibiotic-treated mice, GF mice, MyD88 ${ }^{-1-}$ mice, and Myd8 ${ }^{\text {iAMath1 }}$ mice (Figure 3e). Although CC did not inhibit GAP formation in the SI, luminal EGF could inhibit SI GAPs in SPF-housed mice (Figure 3f), demonstrating that the pathway downstream of the EGFR, regulating GAP mAChR responsiveness and GAP formation in SI GCs, was preserved. In the GF mouse colon, luminal LPS resulted in increased phosphorylation of p42/p44 MAPK and the EGFR, and inhibited GAP formation within minutes (Figure $\mathbf{3 g}, \mathbf{h}$ ). Colonic GAP inhibition in response to LPS was dependent on EGFR phosphorylation (Figure 3h), demonstrating a requirement for EGFR activation downstream of microbial sensing in GAP inhibition in vivo. In addition, EGF inhibition of colonic GAP formation in Abx mice, and SI GAP formation in SPF-housed mice required phosphorylation of p42/p44 MAPK (Figure 3f,i), indicating that the $\mathrm{p} 42 / \mathrm{p} 44 \mathrm{MAPK}$ activation required to suppress GAP formation is downstream of EGFR activation. Together, these observations identify a pathway within colonic GCs where Myd88 signaling activates EGFR, which in turn activates $\mathrm{p} 42 / \mathrm{p} 44$ MAPK and inhibits GC responsiveness to $\mathrm{mAChR}_{4}$ signals and GAP formation.
Antigen-presenting cells (APCs) are recruited to the colonic epithelium and acquire luminal antigen in a $\mathrm{mAChR}_{4^{-}}$ dependent manner when GAPs are present

Studies using reporter mice expressing yellow fluorescent protein (YFP) driven by the CD11c promoter, or CD11 $c^{\mathrm{YFP}}$ reporter mice, ${ }^{20}$ revealed that SI GAPs deliver luminal antigens to the CD11 $\mathrm{c}^{\mathrm{YFP}+}$ LP-DCs associating with the SI epithelium. ${ }^{8}$ In contrast to the situation in the SI, we observed very few $\mathrm{CD} 11 \mathrm{c}^{\mathrm{YFP}+}$ cells residing near the colonic epithelium in SPFhoused mice (Figure $4 \mathbf{a}-\mathbf{c}$ ). Most of the colonic CD11 $\mathrm{c}^{\mathrm{YFP}+}$ cells were deep in the LP, $30 \mu \mathrm{m}$ below the surface. However, in SPF-housed CD11 $\mathrm{c}^{\mathrm{YFP}}$ mice treated $24 \mathrm{~h}$ before imaging with an inhibitor of p42/p44 MAPK (MAPKi) or EGFR (EGFRi) to induce colonic GAPs, a large number of $\mathrm{CD} 11 \mathrm{c}^{\mathrm{YFP}+}$ cells were found immediately below the epithelium (Figure 4a-c). Using flow cytometry to evaluate changes in the LP-DC populations after inducing colonic GAP formation with MAPKi or EGFRi revealed an influx of CD11c + MHCII + cells, $\mathrm{CD} 8{ }^{+} \mathrm{T}$ cells, and $\mathrm{CD} 4{ }^{+} \mathrm{T}$ cells in both the LP and the epithelial fractions of the colon, but not the SI, where GAPs did not increase in response to MAPKi or EGFRi (Figure 4d,e; Supplementary Figures S4 and S5). Following EGFRi treatment increases were seen in the $\mathrm{CD}_{103}{ }^{+} \mathrm{CD}_{11 \mathrm{~b}}{ }^{+} \mathrm{CX}_{3} \mathrm{CR} 1^{-}, \mathrm{CD} 103^{-} \mathrm{CD} 11 \mathrm{~b}+$ $\mathrm{CX}_{3} \mathrm{CR} 1^{\text {int }}$, and $\mathrm{CD} 103+\mathrm{CD} 11 \mathrm{~b}+\mathrm{CX} 3 \mathrm{CR} 1^{\mathrm{hi}} \mathrm{APC}$ subsets, with the largest increase seen in the CD103 + CD11b + CX3CR1 ${ }^{\text {hi }}$ APC subset (Supplementary Figure S5a,b).

To evaluate whether luminal antigen was effectively delivered to colonic LP-APCs when GAPs were present, chicken egg ovalbumin (Ova) was injected into the colonic lumen, and $2 \mathrm{~h}$ later $\mathrm{CD} 103^{+} \mathrm{CD}_{11 \mathrm{c}^{+}} \mathrm{MHCII}^{+}$and $\mathrm{CD} 103^{-} \mathrm{CD} 11 \mathrm{c}^{+}$ $\mathrm{MHCII}^{+}$APCs were isolated from the colonic LP, excluding the colonic patches, and cultured with Ova-specific CD8 ${ }^{+}$ T cells isolated from OTI T-cell receptor transgenic mice. APCs isolated from the colon of vehicle-treated SPF-housed mice given luminal Ova induced no increase in T-cell proliferation when compared with those isolated from mice given luminal phosphate-buffered saline (PBS) (Figure 4f), indicating the antigen is not effectively delivered across the colonic epithelium to LP-APCs in the basal state where GAPs are absent. In contrast, colonic LP-APCs isolated from mice given luminal Ova after treatment with an EGFRi or MAPKi to induce colonic GAPs stimulated significant OTI T-cell proliferation (Figure 4f), demonstrating that luminal antigen was effectively delivered to LP-APCs when GAPs were present. Although luminal antigen was delivered to both $\mathrm{CD}_{103}{ }^{+}$and $\mathrm{CD} 103^{-}$ LP-APCs, luminal antigen was more effectively delivered to colonic $\mathrm{CD}_{103}{ }^{+}$LP-APCs (Figure 4f), similar to what has been reported for antigen delivery by GAPs in the SI. ${ }^{8}$ Consistent with a requirement for GCs in this process, antigen delivery to colonic LP-APCs was not detectable when EGFRi was given to Math $1^{\mathrm{i} \Delta \text { vil }}$ mice treated with RU486 to deplete GCs (Figure 4f). Furthermore, $\mathrm{mAChR}_{4}$ antagonists inhibited luminal Ova delivery to colonic LP-APCs in mice given EGFRi to open colonic GAPs, but did not affect the ability of colonic LP-APCs to stimulate T cells when exogenous Ova was added to the cell culture, indicating that $\mathrm{mAChR}_{4}$ antagonists 

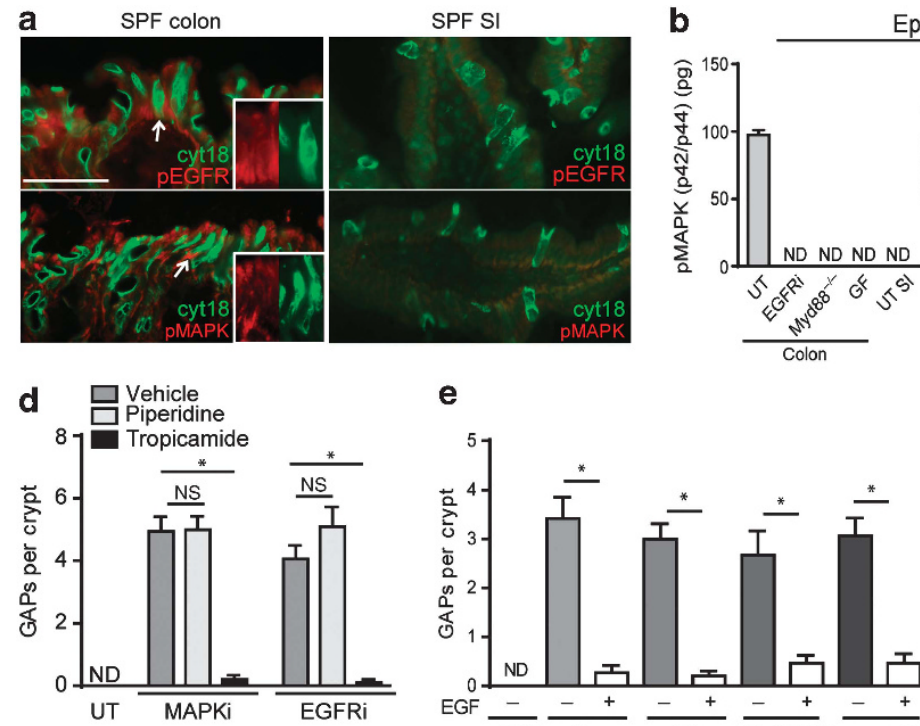

e
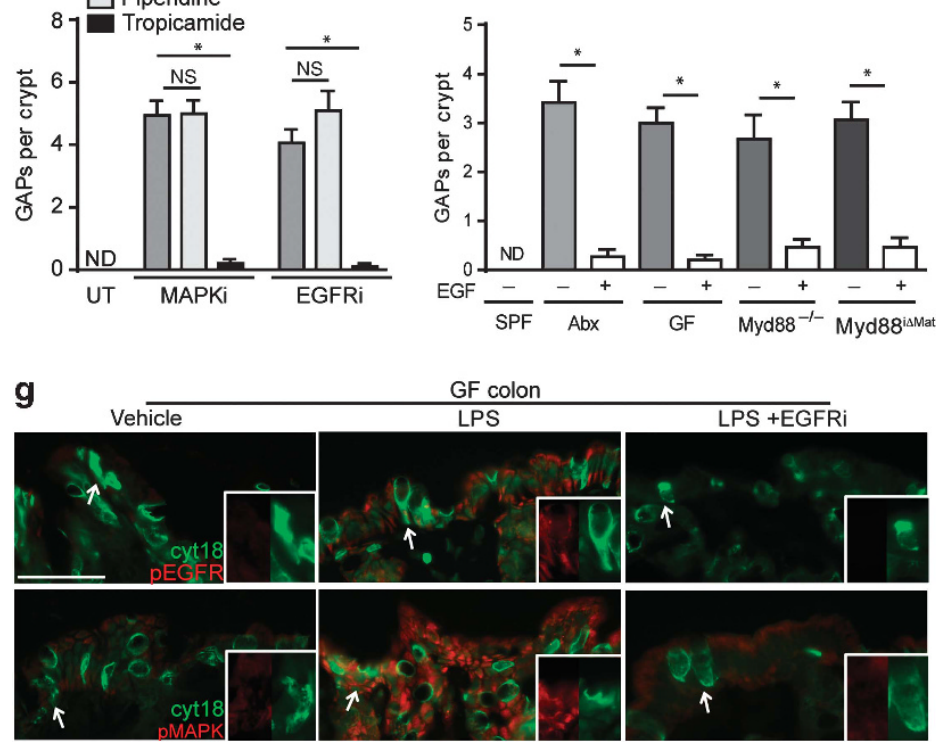

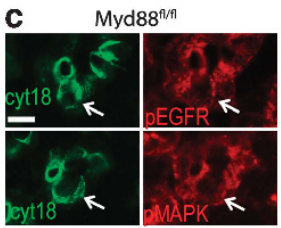

Myd88iMath1

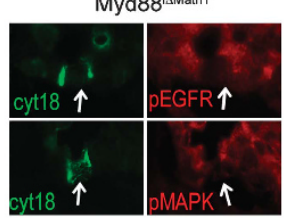

f
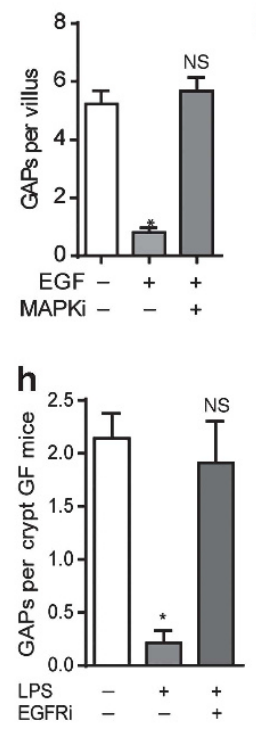

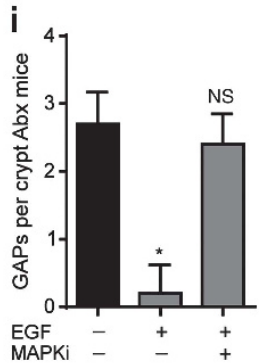

Figure 3 Goblet cell (GC) intrinsic microbial sensing activates the epidermal growth factor receptor (EGFR), p42/p44 mitogen-activated protein kinase (MAPK), and suppresses muscarinic acetylcholine receptor $4\left(\mathrm{mAChR}_{4}\right)$-dependent goblet cell-associated antigen passage (GAP) formation.

(a) Immunofluorescence staining for phosphorylated EGFR (pEGFR: red), phosphorylated p42/p44 mitogen-activated protein kinase (pMAPK: red), and cytokeratin 18 (cyt18: green) in the small intestine (SI) and colon of specific pathogen-free (SPF)-housed mice. White arrow denotes GCs enlarged in inserts with individual channels. (b) Enzyme-linked immunosorbent assays for pMAPK and pEGFR on isolated SI and colonic epithelium from untreated mice (UT), mice treated with an EGFR inhibitor (EGFRi), Myd88 ${ }^{-1-}$ mice, and germ-free (GF) mice. (c) Immunofluorescence for cyt18, pEGFR, and pMAPK in the colon of Myd88 ${ }^{\text {i MMath1 }}$ mice and littermate controls treated with RU486. White arrow indicates the location of a cyt18 + cell in each panel. (d) Number of colonic GAPs per crypt in mice treated with EGFRi or MAPK inhibitor (MAPKi) and antagonists for mAChR $\mathrm{m}_{3}$ and $\mathrm{mAChR}_{4}$. (e) Number of colonic GAPs per crypt from SPF-housed mice with or without antibiotic treatment, GF mice, MyD $88^{-1-}$ mice, and Myd8 $88^{i \Delta M a t h 1}$ mice treated with RU486 and given vehicle or luminal EGF. (f) Number of SI GAPs per villus in SPF-housed mice treated with EGF and MAPKi. (g) Immunofluorescence for cyt18 (green), pEGFR (red), and pMAPK (red) in the colon of GF mice following intraluminal vehicle (left panel), lipopolysaccharides (LPS) (middle panel), or LPS and intraperitoneal EGFRi treatment (right panel). White arrows denote GCs enlarged in insets with individual channels. (h) Number of GAPs per crypt from the colon of GF mice treated with LPS with and without EGFRi treatment. (i) Number of GAPs per crypt from the colon of antibiotic-treated mice given EGF with and without MAPKi treatment. Data are presented as the mean \pm s.e.m., ${ }^{\star} P<0.05$. ND, not detected; NS, not significant. $n=3$ or more mice for each condition in panels $\mathbf{a}, \mathbf{b}$, and $\mathbf{d}-\mathbf{i}$, panel $\mathbf{c}$ is representative of one of two mice for each condition. Scale bar $=20 \mu \mathrm{m}$ in panels $\mathbf{a}$ and $\mathbf{g}$; scale $\mathrm{bar}=5 \mu \mathrm{m}$ in panel $\mathbf{c}$.

inhibited delivery of antigen to LP-APCs and GAP formation, but did not affect the antigen presentation capacity of LP-APCs (Figure 4g).

\section{Overriding GC intrinsic microbial sensing and inappropriately opening colonic GAPs results in the recruitment of APCs and neutrophils and the production of inflammatory cytokines}

The suppression of colonic GAPs by microbial sensing in the healthy basal state suggests that exposure of the LP immune system to substances from the colonic lumen is normally avoided, and that exposure to colonic luminal contents may have untoward outcomes despite the absence of overt patho- gens in the commensal microbiota. To evaluate the effect of prolonged opening of colonic GAPs in the setting of the normal commensal flora and intact microbial sensing by the immune system, we treated Myd88 ${ }^{\mathrm{i} \Delta \text { Math1 }}$ mice or their littermates, $\mathrm{Myd} 88^{\mathrm{fl} / \mathrm{fl}}$ mice, with sequential injections of RU486 over the course of 4 days and evaluated the LP-DC populations and antigen delivery in the colon. Similar to mice treated with EGFRi or MAPKi, changes in the LP-APC populations were also observed in Myd88 ${ }^{\text {iAMath1 }}$ mice lacking Myd88 in GCs (Figure 5a,b). However, there was not an increase in colonic LP-APCs in mice lacking Myd88 globally (Figure 5b) or in EGFRi- or MAPKi-treated mice given antibiotics (Figure 5c), implicating a role for Myd88-dependent micro- 


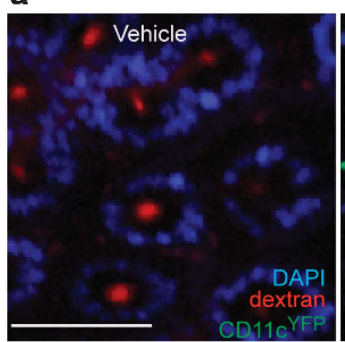

C

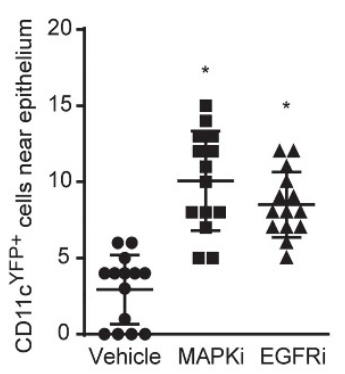

f
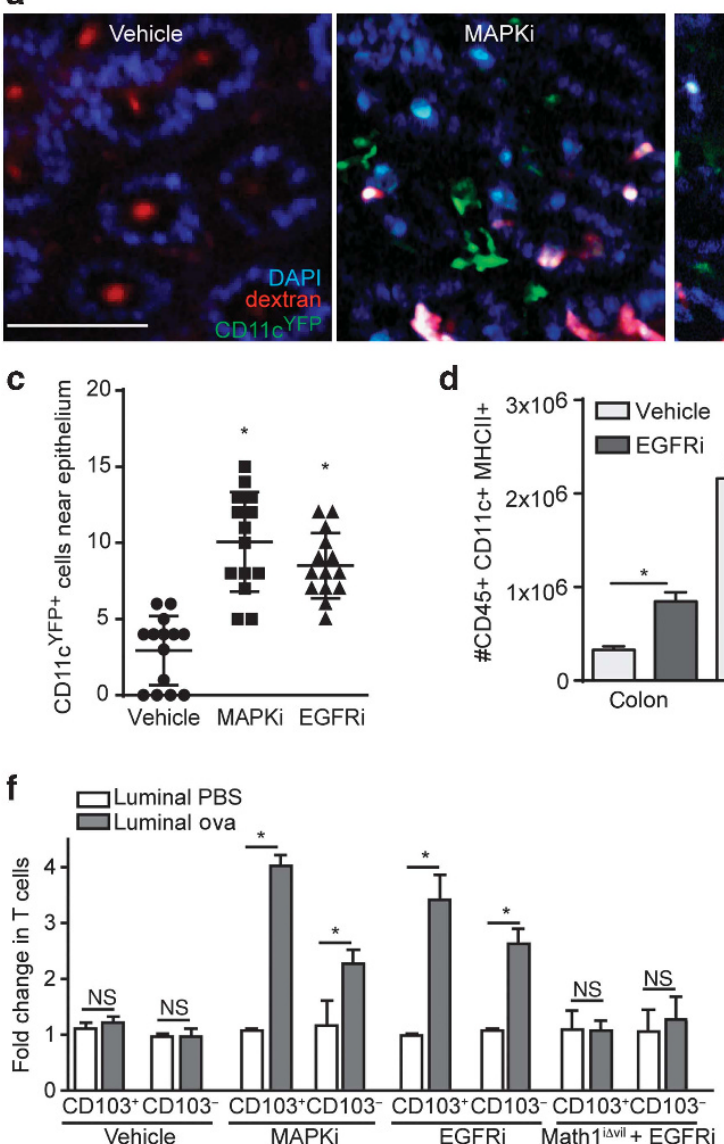

d b
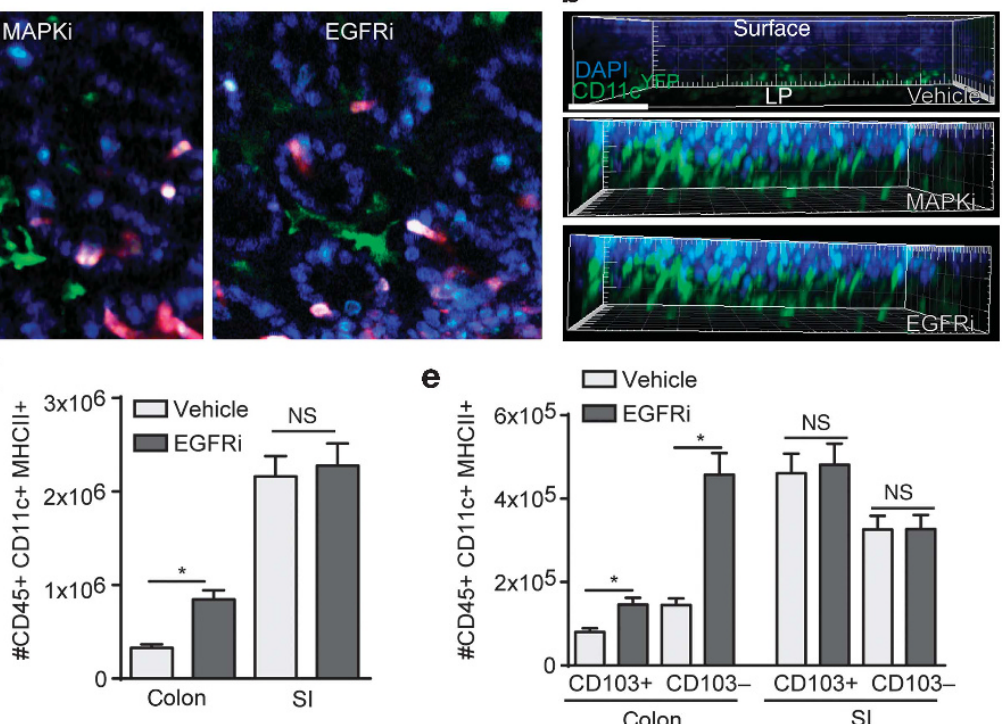

e

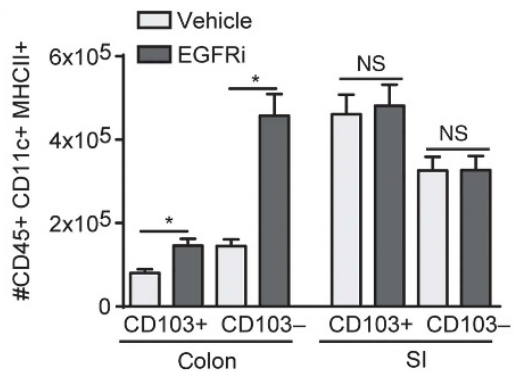

g

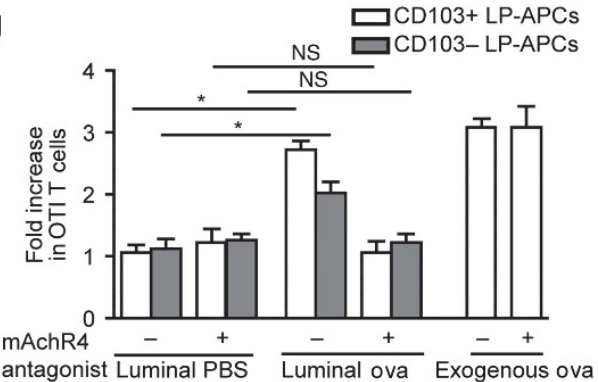

Figure 4 Antigen-presenting cells (APCs) are recruited to the colonic epithelium and acquire luminal antigen in a muscarinic acetylcholine receptor 4 $\left(\mathrm{mAChR}_{4}\right)$-dependent manner when goblet cell-associated antigen passages (GAPs) are present. (a) Two-photon images of the colon $20 \mu \mathrm{m}$ below the surface following the administration of luminal dextran (red) and diamidino-2-phenylindole (DAPI) (blue) in specific pathogen-free (SPF)-housed $\mathrm{CD} 11 \mathrm{c}^{\mathrm{YFP}}$ reporter (green) mice treated $24 \mathrm{~h}$ earlier with vehicle (left panel), p42/p44 mitogen-activated protein kinase inhibitor (MAPKi) (middle panel), or epidermal growth factor receptor inhibitor (EGFRi) (right panel). (b) Three-dimensional reconstruction of $Z$-stacks from the treatment groups with the red channel removed to better identify CD11c $\mathrm{C}^{\mathrm{YFP}}+$ cells (major ticks $=20 \mu \mathrm{m}$ ). (c) The number of CD11c $\mathrm{c}^{\mathrm{YFP}+}$ cells in each group in a volume of $250 \mu \mathrm{m}(x) \times 220 \mu \mathrm{m}(y) \times 20 \mu \mathrm{m}(z)$ obtained from the colonic epithelial surface extending into the lamina propria (LP) in the $z$-plane. (d) Number of

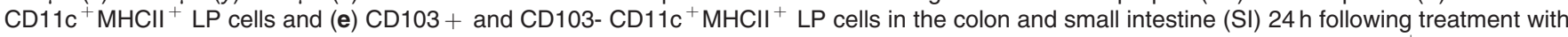
vehicle or EGFRi as assessed by flow cytometry. ( $\mathbf{f}$ and $\mathbf{g}$ ) Luminal antigen acquisition and presentation capacity of colonic $\mathrm{CD}^{-103^{+}}$and $\mathrm{CD} 103^{-}$ LP-APCs isolated from (f) vehicle-treated, MAPKi-treated, or EGFRi-treated SPF-housed mice or EGFRi-treated goblet cell-depleted Math ${ }^{i \Delta v i l}$ mice or isolated from (g) SPF-housed mice treated with EGFRi and $\mathrm{mAChR}_{4}$ antagonists following the administration of luminal phosphate-buffered saline (PBS) or ovalbumin (Ova). Luminal antigen acquisition and presentation capacity was assessed by the expansion of Ova-specific OTI T cells following $72 \mathrm{~h}$ of culture. Data are presented as the mean \pm s.e.m., ${ }^{\star} P<0.05$. MHC, major histocompatibility complex; ND, not detected; NS, not significant. $n=3$ or more mice for each condition in panels a-e, data in panels $\mathbf{f}$ and $\mathbf{g}$ are representative of one of three replicates generated by pooling LP-APCs from two mice in each group. Scale bar $=50 \mu \mathrm{m}$.

bial sensing in cell types other than GCs in recruiting cellular populations in the colon when GAPs were present. Confirming antigen delivery occurs in the colon of mice lacking Myd88 in GCs, colonic APCs isolated from Myd88 ${ }^{\mathrm{i} \triangle \mathrm{Math} 1}$ mice, and not those isolated from Myd88 $8^{\mathrm{fl} / \mathrm{fl}}$ littermate mice, given RU486 and luminal Ova induced T-cell proliferation in ex vivo assays (Figure 5d).

Myd8 $8^{\text {i } \triangle \text { Math1 }}$ mice with colonic GAPs opened for 4 days had significantly elevated production of interleukin (IL)-6 in the mesenteric lymph node and colonic LP and significantly elevated IL-17 in the colonic LP with a trend toward elevated production of IL-6 in the serum (Figure 5e). We also observed elevated production of the neutrophil chemoattractant CXCL1 and an influx of neutrophils in the colonic mucosa (Figure $\mathbf{5 f}-\mathbf{i}$ ) indicative of an acute inflammatory response. Therefore, inhibiting GC intrinsic microbial sensing and inappropriately delivering luminal contents to the normal colonic immune system resulted in an inflammatory response in the absence of an overt pathogenic infection.

\section{DISCUSSION}

The primary function of the non-follicle-bearing epithelium of the gastrointestinal tract is absorption of nutrients, requiring that this be a single-layer expansive epithelial surface that is exposed to the environment. In addition to absorption, the epithelium also forms a barrier protecting the host from 

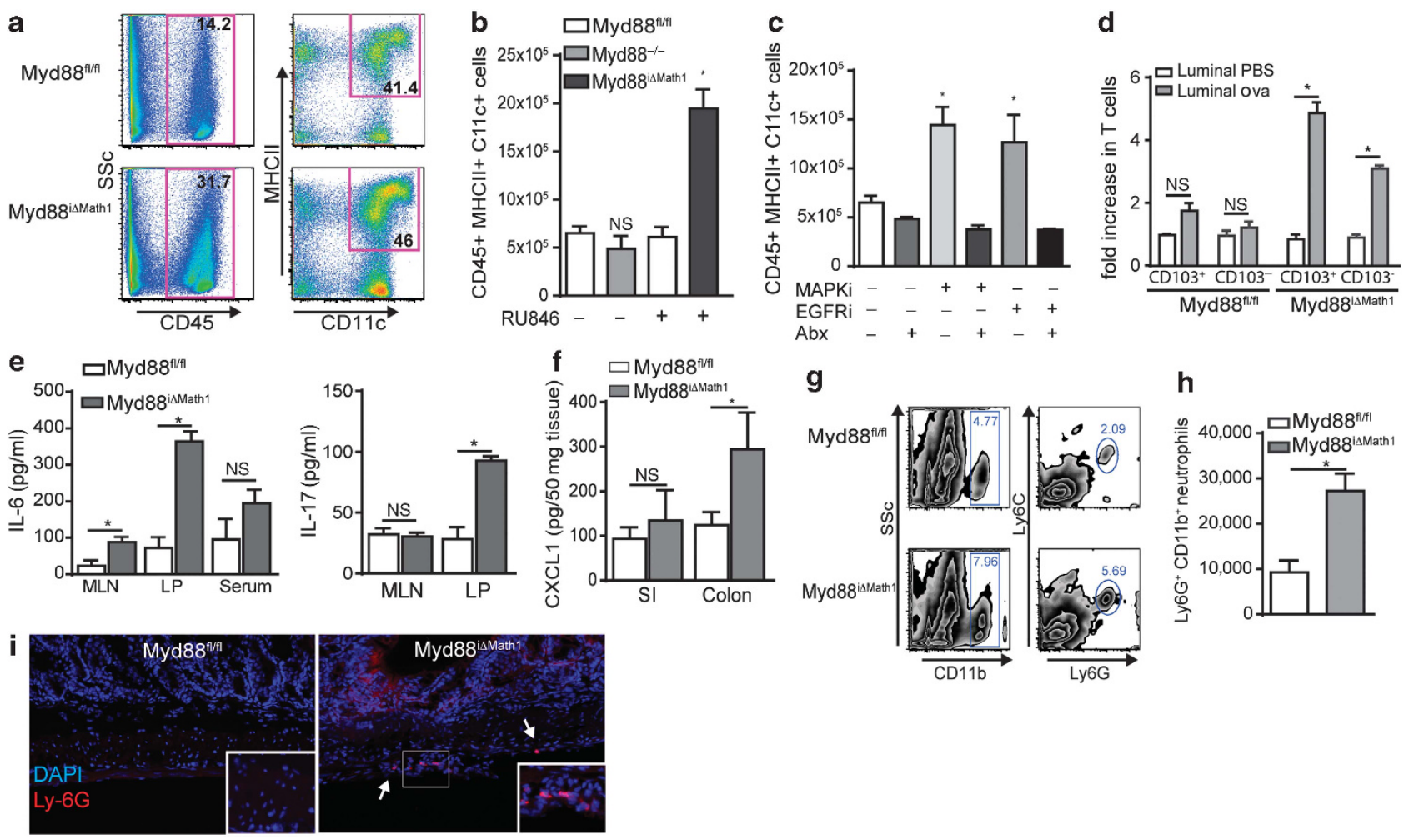

Figure 5 Overriding goblet cell intrinsic microbial sensing and opening colonic goblet cell-associated antigen passages results in an influx of leukocytes and inflammatory cytokine production in response to a non-pathogenic commensal microbiota. (a) Representative flow plots of CD45 + CD11c + $\mathrm{MHCll}+$ cells in Myd88 ${ }^{\mathrm{i} \Delta M a t h 1}$ mice and Myd8 ${ }^{\mathrm{fl} / \mathrm{fl}}$ littermates treated with RU486. (b and c) Number of CD45 + CD11c + MHCII + LP cells as assessed

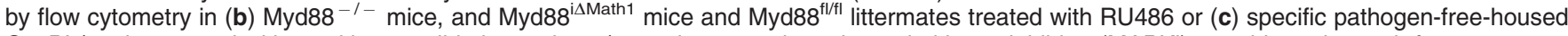
C57BL/6 mice treated with or without antibiotics and p42/p44 mitogen-activated protein kinase inhibitor (MAPKi) or epidermal growth factor receptor inhibitor (EGFRi). (d) Luminal antigen acquisition and presentation capacity of colonic CD103 ${ }^{+}$and CD103 ${ }^{-}$LP-antigen-presenting cell isolated from

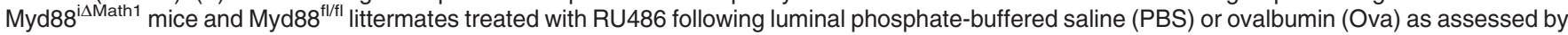
the expansion of Ova-specific OTI T cells following $72 \mathrm{~h}$ of culture. (e) Interleukin (IL)-6 (left panel) and IL-17 (right panel) levels in the serum and culture supernatants from mesenteric lymph node (MLN) or colonic LP cells and (f) CXCL1 levels in small intestine (SI) or colon tissue homogenates from

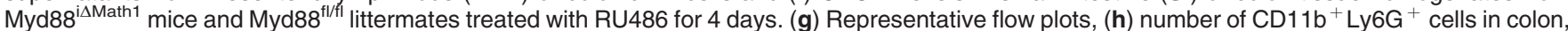

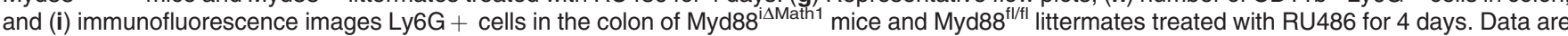
presented as the mean \pm s.e.m., ${ }^{*} P<0.05$. DAPI, diamidino-2-phenylindole; LP, lamina propria; MHC, major histocompatibility complex; ND, not detected; NS, not significant; Ssc, side scatter. $n=3$ or more mice for each condition. Scale bar $=50 \mu \mathrm{m}$.

potential pathogens. Furthermore, combining observations from multiple studies implicates the non-follicle-bearing epithelium as a site of immune surveillance, which is important for homeostatic responses to innocuous antigens, such as tolerance to dietary antigens, and for immunity to pathogens. ${ }^{2,21-23}$ How the epithelium and the underlying immune system within the LP simultaneously perform these diverse functions of barrier maintenance, absorption, and immune surveillance has been a question of significant interest in mucosal immunology.

Although multiple pathways delivering macromolecular substances from the lumen across the intestinal epithelium have been identified, the relative importance of each pathway in LP immune surveillance different settings and how each pathway is regulated to control access to luminal substances to the immune system are largely uninvestigated. The epithelial layer of the gastrointestinal tract maintains its protective barrier in part through the formation of tight junctions between epithelial cells, which can allow luminal substances to cross the epithelium via a pore formed by claudin proteins that allows small solutes to pass, ${ }^{24}$ and via the paracellular leak pathway that allows larger macromolecules, including proteins and carbohydrates, to cross the epithelial barrier. ${ }^{25}$ Paracellular leak was reported to be present in mice lacking GCs, but luminal antigen was not effectively delivered to SI LP-DCs as assessed by their inability to induce antigen-specific T-cell responses, ${ }^{8}$ suggesting that paracellular leak may not effectively deliver antigen to LP-DCs in the steady state. Alterations in tight junctions and paracellular leak resulting in increased flux across the epithelium have been associated with a predisposition to develop inflammation and overt inflammation, ${ }^{25,26}$ suggesting that this pathway could be an important contributor to disease processes. Therefore, although the immune outcomes downstream of antigen delivery via paracellular leak are not well understood, observations suggest this pathway may not have a major role in steady-state immune surveillance.

Microfold (M) cell-mediated transcytosis is the most studied mechanism delivering antigen across the intestinal epithelium 
to the immune system. The vast majority of $\mathrm{M}$ cells are found as part of the follicle-associated epithelium overlying lymphoid follicles, with rare $\mathrm{M}$ cells located in the villous epithelium in the SI steady state. ${ }^{27,28}$ Current understanding is that $\mathrm{M}$ cells continuously deliver antigens to the underlying immune system irrespective of the types of antigen and environmental stimuli, suggesting that regulation of antigen delivery to the LP via $\mathrm{M}$ cells would require altering the number of villous $\mathrm{M}$ cells. Villous M-cell numbers can increase in number in response to environmental stimuli, including exogenous toxins and endogenous cytokines; ${ }^{28,29}$ however, this requires days, indicating that regulating antigen delivery via $M$ cells in this manner is not rapid. Moreover, the rarity of villous $\mathrm{M}$ cells in the SI and their apparent absence in the non-follicle-bearing epithelium in the colon, suggests that villous $\mathrm{M}$ cells would be minor contributors to steady-state LP immune surveillance.

Multiple studies have described the ability of LP-DCs to pass dendrites between epithelial cells and extend them into the lumen to acquire luminal substances and bacteria. ${ }^{30-34}$ When observed, LP-DC trans-epithelial dendrites (TEDs) have largely been noted in the SI as opposed to the colon. Similar to our findings here, regarding GC-mediated antigen delivery, epithelial Myd88-dependent sensing of the microbiota regulated TED formation by LP-DCs, ${ }^{32}$ and accordingly would regulate luminal antigen access to the LP immune system. However, in contrast to our findings of antigen delivery by colonic GCs, TED formation by LP-DCs was induced, ${ }^{32}$ not inhibited by the microbiota. These observations demonstrate reciprocal regulation of antigen access to the LP immune system by GCs and LP-DC TED formation, and imply different contributions of these antigen delivery mechanisms. The microbial stimuli regulating antigen delivery need not be pathogenic, as the commensal microbiota can inhibit colonic GAPs and LP-DC TED formation has been observed in the absence of enteric infection. ${ }^{31,32}$

Recently, it was discovered that when GCs secrete they can form GAPs and deliver luminal antigens to LP-DCs in the SI, ${ }^{8}$ suggesting that this could be a mechanism whereby the epithelium allows immune surveillance of the luminal contents while simultaneously maintaining the barrier with mucus secretion. The ability to augment GAPs and antigen delivery by agents inducing GC mucous secretion, the dynamic nature of GAPs, appearing and disappearing during the imaging window, and the lack of GAPs in the colon despite the presence of abundant GCs, ${ }^{8}$ suggested that GAP formation and antigen delivery could be regulated. In addition, Mucin 2, a GCderived component of mucus, was found to imprint DCs with tolerogenic signals ${ }^{35}$ suggesting that when GCs secrete they can form GAPs to deliver luminal antigens in the context of tolerogenic signals to DCs. Here we identify that in the basal state, SI GAP formation is driven by ACh acting on the $\mathrm{mAChR}_{4}$. Notably, cholera toxin, a well-known GC secretagogue, did not induce GAP formation, demonstrating that not all stimuli inducing GC secretion induce GAPs. GCs are known to secrete by two pathways, compound exocytosis, an intracellular $\mathrm{Ca}^{2+}$-driven pathway in which multiple vesicles of mucin fuse and are secreted, and primary exocytosis, a cyclicadenosine-monophosphate-driven pathway in which single mucin vesicles fuse with the plasma membrane during secretion. ${ }^{9,36-38}$ The association of GAP formation with Ach-induced secretion, which occurs by compound exocytosis, but not with cholera toxin-induced secretion, which occurs by primary exocytosis, suggests that GAP formation could be linked with specific types of GC secretion. This may allow GCs to secrete mucus to maintain the barrier and not form GAPs to deliver luminal antigens in situations where antigen delivery to the immune system could be unfavorable.

Colonic GCs were unresponsive to ACh to form GAPs and deliver antigen to the colonic LP-APCs, when the luminal microbiota and GC intrinsic microbial sensing were intact. Disruption of either the microbiota or of GC microbial sensing relieved the repression of the $\mathrm{mAChR}_{4}$ pathway in colonic GCs and induced GAPs to form, which allowed luminal antigen delivery to both $\mathrm{CD} 103^{+}$and $\mathrm{CD} 103^{-}$colonic LP-APCs, with more effective delivery to CD103 + colonic LP-APCs. This could suggest that both LP-APC populations interact with the colonic GAPs, with the CD103 + LP-APCs interacting with GAPs more frequently.

Recently, Mazzini et al. reported a critical role for gap junction-dependent antigen transfer between SI LP-DCs in the acquisition of luminal antigen. ${ }^{39}$ Similar to the report describing SI GAPs, ${ }^{8}$ Mazzini et al. ${ }^{39}$ found that luminal antigen was only effectively delivered to CD103 + LP-DCs as assessed by the ability to induce antigen-specific T-cell responses. Moreover, antigen delivery to CD103 + LP-DCs was found to be dependent on the DC expression of the gap junction protein connexin 43 in one study, and on GCs in the other, as deletion of either connexin 43 in DCs, or deletion of GCs, via deletion of Math1 in intestinal epithelial cells, abolished luminal antigen delivery to SI CD103+ LP-DCs. ${ }^{8,39}$ Of note, gap junction proteins, including connexin 43 , are expressed by GCs, as determined by gene expression microarrays. These observations raise the possibility that these pathways may be related.

Shutting down colonic GAPs by microbial ligands or opening up colonic GAPs by manipulating microbial sensing or the downstream EGFR p42/p44 MAPK pathway occurred rapidly. Heat-killed CC and TLR ligands were sufficient to inhibit GAP formation, indicating that live bacteria are not required for this process and suggesting GCs may sense microbial products that can gain access to the epithelium through the mucus layer, which acts as a physical barrier preventing whole bacteria from contacting the epithelium in the colon. ${ }^{40}$ In combination with the GC intrinsic nature of these events, these observations indicate that regulation of antigen delivery by GAPs is rapid and local, and that colonic GCs are active participants in immune surveillance by controlling the immune system's access to luminal antigens.

Interestingly, we observed that SI GAPs were not rapidly shut down in response to heat-killed CC and they were unchanged in mice lacking Myd88 in GCs, indicating that SI GCs were relatively unresponsive to microbial ligands. SI GCs expressed 
lower levels of TLRs and higher levels of inhibitors of TLR signaling, as seen by gene expression microarrays, which could explain their relative unresponsiveness to microbial ligands and suggests that GC sensing of microbial stimuli does not have a major role suppressing SI GAPs in the normal steady state. Although the SI expresses similar levels of EGFR, phosphorylated EGFR could not be detected, demonstrating that unlike the colon, EGFR activation in SI GCs is absent at the steady state. However, SI GAPs could still be shut off with exogenous EGF, through activation of $\mathrm{p} 42 / \mathrm{p} 44 \mathrm{MAPK}$, indicating that similar regulatory pathways of $\mathrm{mAChR}_{4}$ responsiveness are present in SI GCs. The significance of retaining the pathways downstream of the EGFR that inhibit $\mathrm{mAChR}_{4}$ responses in SI GCs, in the setting of the relative inability of microbial ligands to shut off SI GAPs is unclear; however, it could suggest that in some situations, such as enteric infection, SI GCs may alter their responsiveness to microbial stimuli, potentially via increased TLR expression or decreased expression of inhibitors of TLR signaling, and shut off GAPs similar to colonic GCs. A further implication of these observations is that in the steady state, luminal antigen delivery to the SI LP-APCs is largely regulated by $\mathrm{ACh}$ production and release, which is predominantly neuronal in origin, suggesting situations in which the presence or absence of antigen delivery to the SI LP could be linked to environmental stimuli inducing neuronal production of ACh.

The absence of GAPs and antigen delivery to LP-APCs, combined with the spatial separation of LP-APCs from the colonic epithelium we observed in vivo in healthy wild-type SPF-housed mice, suggests that in the steady state the colonic LP immune system is relatively unaware of the colonic luminal contents. Immune surveillance in this situation may primarily occur through the follicle-associated epithelium overlying the colonic patches and lymphoid follicles. GCs have been reported in the follicle-associated epithelium, ${ }^{41}$ and rare GAPs have been noted on the periphery of the follicle-associated epithelium overlying the cecal path in the steady state. ${ }^{8}$ How these are regulated and whether these contribute to immune surveillance remains to be explored. Intrinsic differences in the function of the SI and colon could justify the difference in antigen delivery and interactions of APCs with the epithelium between the two organs, with the SI actively absorbing dietary nutrients, which the immune system may survey to induce tolerance, and the colon largely absorbing water and solutes, which are not readily recognized by the adaptive immune system. This difference could also be attributed to the more abundant and complex microbial stimuli in the colon, which makes recognizing and appropriately responding to innocuous antigens in this environment tenuous. In support of the latter possibility, we observed that opening colonic GAPs for 4 days resulted in the production of inflammatory cytokines and chemokines and an influx of leukocytes including neutrophils, indicative of acute inflammation, despite the lack of overt pathogens in the commensal microbiota. This suggests that the lack of antigen delivery to the colonic LP immune compartment is not primarily to promote ignorance or disinterest, but to avoid inappropriate responses by the colonic LP immune system to luminal antigens encountered in a complex microbial environment.

The intestinal epithelium performs the diverse and essential functions of absorption, barrier maintenance, and immune surveillance. How the immune system gains access to luminal antigens without compromising the epithelial barrier and absorption, and how the immune system appropriately responds to innocuous antigens encountered in a complex environment of abundant microbiota and microbial stimuli are central questions in intestinal immunology. Here, we identify the stimuli and pathways regulating GAP formation and luminal antigen delivery to LP-APCs in the steady state, and further link this process with GC secretion. These observations demonstrate novel ways in which GCs contribute to diverse epithelial functions and uncover a central role for colonic GC sensing of the luminal microbiota to regulate the immune system's exposure and access to luminal substances.

\section{METHODS}

Mice. All mice used in these studies were on the C57BL/6 background. C57BL/6 mice were purchased from The National Cancer Institute (Frederick, MD) or The Jackson Laboratory (Bar Harbor, MN). OTI T-cell receptor transgenic mice, ${ }^{42}$ Math $1^{\mathrm{fl} / \mathrm{fl}}$ mice, ${ }^{13}$ Math $1^{\text {PGRCre }}$ mice, ${ }^{43} \mathrm{CD} 11 \mathrm{c}^{\mathrm{YPP}}$ transgenic mice, ${ }^{20} \mathrm{CX}_{3} \mathrm{CR} 1^{\mathrm{GFP}}$ mice, ${ }^{44}$ and ROSA $^{\text {dtomato }}$ mice ${ }^{45}$ were purchased from The Jackson Laboratory. Myd88 ${ }^{-1-}$ mice, ${ }^{46}$ a gift from Dr Akira (Osaka University, Osaka, Japan), were bred onto the C57BL/6 background by the Speed Congenics Facility of the Rheumatic Diseases Core Center, and maintained in house. Transgenic mice bearing a tamoxifen-dependent Cre recombinase expressed under the control of the villin promoter (vil-Cre-ERT2) mice ${ }^{47}$ were a gift from Sylvie Robine (Institut Curie, Paris, France). Math $1^{\mathrm{fl} / \mathrm{fl}}$ mice were bred to vil-Cre-ERT2 mice to generate mice with inducible depletion of GCs following deletion of Math1 in villin-expressing cells, referred to as Math $1^{\mathrm{i} \Delta \mathrm{vil}}$ mice. To deplete GCs, Math $1^{\text {i } \Delta \text { vil }}$ mice were injected with $100 \mu \mathrm{g}$ tamoxifen (Sigma-Aldrich, St Louis, MO) dissolved in sunflower seed oil with $20 \%$ ethanol (Sigma-Aldrich) intraperitoneally (i.p.) daily for 4 days. Mice were used for experiments 3 or more days after the last injection. In some experiments, mice were placed on antibiotics for 1 week and then analyzed for GAP formation by two-photon microscopy as described below. Colon tissue was sectioned and stained for GCs with alcian blue to ensure GCs were knocked out. To generate Math1 reporter mice, Math ${ }^{\text {PGRCre }}$ mice, which express a progesteroneinducible Cre under the control of the Math1 locus, were bred with ROSA $^{\text {dtomato }}$ mice, referred to as dtom ${ }^{\mathrm{iMath} 1}$ mice. To label colonic GCs, dtom ${ }^{\text {iMath1 }}$ mice were injected with $200 \mu \mathrm{g}$ RU486 (mifepristone, Caymen Chemicals, Ann Arbor, MI) dissolved in sesame oil (Acros Organics, Fair Lawn, NJ) i.p. every day for 7 days. To delete Myd88 signaling from GCs, Myd8 $8^{\mathrm{fl} / \mathrm{fl}}$ mice were bred to Math $1^{\text {PGRcre }}$ mice, referred to as to Myd88 $8^{\text {i } \Delta \text { Math } 1}$ mice. To induce deletion of Myd88 in GCs, Myd $88^{\text {i } \Delta \text { Math } 1}$ mice were injected with $200 \mu \mathrm{g}$ RU486 i.p. on day 0 and day 2 , and analyzed on day $4 . \mathrm{Myd} 88^{\mathrm{fl} / \mathrm{fl}} \mathrm{Math} 1{ }^{\mathrm{WT}}$ littermate mice injected with RU486 were used for controls. Gnotobiotic mice were obtained from the Washington University Digestive Disease Research Core Center murine models core. Animals, other than gnotobiotic mice, were housed in a specific pathogen-free facility and fed routine chow diet. In some experiments, mice were treated with a combination of antibiotics (Abx); ampicillin ( $1 \mathrm{~g} / \mathrm{l})$, metronidazole $(1 \mathrm{~g} / \mathrm{l})$, neomycin $(1 \mathrm{~g} / \mathrm{l})$, and vancomycin $(500 \mathrm{mg} / \mathrm{l})$ in drinking water for 10 days. Animals were 6-16 weeks of age at the time of analysis. Animal procedures and protocols were carried out in accordance with the institutional review board at the Washington University School of Medicine. 
Isolation of cellular populations and flow cytometric sorting. SIs were harvested, rinsed with PBS and Peyer's patches were removed. Epithelium-associated DCs and T cells were released by incubating for 15 min two times in a $37-{ }^{\circ} \mathrm{C}$ rotating incubator in Hanks' balanced salt solution (HBSS) media (BioWhittaker, Walkersville, Maryland) containing $5 \mathrm{~mm}$ EDTA as previously described. ${ }^{48}$ After a third incubation with HBSS media with $5 \mathrm{~mm}$ EDTA, isolation of splenic and LP cellular populations was performed as previously described. ${ }^{48}$ Cellular suspensions were then passed through a $50-\mu \mathrm{m}$ nylon filter and the cells were prepared for flow cytometric analysis or sorting. Antibodies used for analysis are listed in Supplementary Table S1. DC subpopulations in the SI or APCs in the colon were identified as $7 \mathrm{AAD}^{-}, \mathrm{CD}_{4} 5^{+}, \mathrm{CD} 11 \mathrm{c}^{+}, \mathrm{MHCII}^{+}$, and either $\mathrm{CD} 103^{+}$or $\mathrm{CD}_{103}{ }^{-}$for flow cytometric sorting. To isolate GCs, small intestinal or colonic epithelium was released by incubating for $15 \mathrm{~min}$ in a $37-{ }^{\circ} \mathrm{C}$ rotating incubator in HBSS media (BioWhittaker) containing $5 \mathrm{~mm}$ EDTA as previously described. GCs were identified as CD45 $\mathrm{CD} 24^{-} \mathrm{CK}-18^{+} \mathrm{UEA}^{-} 1^{+}$, whereas IECs were identified as CD $45^{-}$ CD24 ${ }^{-}$CK- $18^{-}$UEA- $1^{-}$and sorted directly into RLT buffer (Qiagen, Valencia, CA) for RNA extraction.

Immunohistochemistry. Immunohistochemistry on frozen sections was performed as previously described. ${ }^{48}$ Antibodies used for immunohistochemistry are listed in Supplementary Table S1. Pseudo-colored black and white images from fluorescent microscopy were obtained with an axioskop 2 microscope (Carl, Zeiss Microscopy, Thornwood, NY) using Axiovision software (Carl Zeiss MicroImaging, Thornwood, NY).

Intravital two-photon microscopy. Mice were anesthetized using nebulized isofluorane in $95 \% \mathrm{O}_{2} / 5 \% \mathrm{CO}_{2}$. Intravital preparation of the SI was performed as previously described. ${ }^{8}$ Briefly, a small midline vertical incision was made through the skin and peritoneum revealing the intestine. To image from the luminal surface, a $7-\mathrm{mm}$ incision was made to expose the luminal surface of the SI. Large fecal particles were removed with care to prevent damaging the luminal surface. Smaller fecal particles were more difficult to remove, and in order to avoid disrupting the mucous layer and the epithelia, were left in place. Imaging was performed with the intestine remaining within the peritoneal cavity. Fluorescently labeled dextran 10,000 MW $(10 \mathrm{mg} / \mathrm{ml}$, Invitrogen, Carlsbad, CA $)$ and diamidino-2-phenylindole $(10 \mathrm{mg} / \mathrm{ml}$, Invitrogen) were injected intraluminally as described previously ${ }^{8}$ to identify GAPs. Tissues were excited using a Ti:sapphire laser tuned to $890 \mathrm{~nm}$ (Chameleon XR, Coherent, Santa Clara, CA). In some experiments mice were given the following treatments before intraluminal dextran administration: $600 \mathrm{mg} / \mathrm{kg}$ atropine chloride (Sigma-Aldrich) i.p $2 \mathrm{~h}$ prior, $150 \mathrm{mg} / \mathrm{kg}$ CCh (Sigma-Aldrich) i.p $10 \mathrm{~min}$ prior, $100 \mathrm{mg} / \mathrm{kg}$ tropicamide or $100 \mathrm{mg} / \mathrm{kg}$ 4-DAMP, $\left(\mathrm{mAChR}_{4}\right.$-selective antagonists, Sigma-Aldrich) i.p. $20 \mathrm{~min}$ prior, $100 \mathrm{mg} / \mathrm{kg}$ piperidine or $100 \mathrm{mg} / \mathrm{kg}$ himbacine, $\left(\mathrm{mAChR}_{3}\right.$-selective antagonists, Sigma-Aldrich) i.p. $20 \mathrm{~min}$ prior, $500 \mu \mathrm{g} / \mathrm{kg}$ tryphostin AG1478 (inhibitor of EGFR phosphorylation, Sigma-Aldrich) i.p. $20 \mathrm{~min}$ prior, $10 \mu \mathrm{g}$ murine recombinant EGF (Shenandoah Biotechnologies, Warwick, PA) injected intraluminally $20 \mathrm{~min}$ prior, $10 \mu \mathrm{g}$ LPS (Sigma-Aldrich) injected intraluminally $20 \mathrm{~min}$ prior, or $10 \mathrm{mg} / \mathrm{kg} \mathrm{U}-0126$ (inhibitor of p42/p44 MAPK phosphorylation, Sigma-Aldrich) i.p. $20 \mathrm{~min}$ prior. CCs from a SPF-housed mouse was resuspended in $1 \mathrm{ml} \mathrm{PBS}$ and heat-killed at $95^{\circ} \mathrm{C}$ for $30 \mathrm{~min}$, and $50 \mu \mathrm{l}$ of this suspension was injected intraluminally $20 \mathrm{~min}$ before dextran administration. After imaging, tissue was fixed with $10 \%$ formalinbuffered solution for 30 min before Optimal Cutting Temperature Compound (Fisher Scientific, Pittsburgh, PA) embedding.

Enumeration of GAPs. GAPs were identified as dextran-filled columns measuring $\sim 20 \mu \mathrm{m}$ (height) $\times 5 \mu \mathrm{m}$ (diameter) traversing the epithelium and containing a nucleus. When present, colonic GAPs were found in all regions of the colon examined, both on the surface epithelium and within the crypts. GAPs were enumerated in the jejunum or mid-transverse colon using Z-stack images of a volume of $225 \mu \mathrm{m}(x)$ by $250 \mu \mathrm{m}(y)$ by $50 \mu \mathrm{m}(z)$ acquired during a 20 -min imaging window beginning $20 \mathrm{~min}$ after dextran administration. SI GAPs were enumerated as GAPs per villus, and colonic GAPs were enumerated within the crypts as GAPs per crypt, which were identified as a ring of diamidino-2-phenylindole-positive epithelial nuclei with fluorescent dextran in the lumen (center). The number of GAPs per villus and GAPs per crypt seen by two-photon imaging were confirmed by immunofluorescence microscopy of tissues that were fixed following two-photon imaging.

Ex vivo analysis of effect of TLR ligands on GAP formation. Colonic tissue from germ-free-housed mice were cut into $2-\mathrm{cm}$ pieces and placed in a 48 -well culture plate containing $500 \mu \mathrm{l}$ media containing $10 \%$ fetal bovine serum, with fluorescent dextran 10,000 MW $(10 \mathrm{mg} / \mathrm{ml})$, and, in some conditions, $10 \mu \mathrm{g} / \mathrm{ml} \mathrm{LPS}, 10 \mu \mathrm{g} / \mathrm{ml} \mathrm{LTA}$, or $10 \mu \mathrm{g} / \mathrm{ml}$ PolyI:C, and incubated for $30 \mathrm{~min}$ at $37^{\circ} \mathrm{C}$. Tissue was fixed with $10 \%$ formalin-buffered solution for $30 \mathrm{~min}$ before Optimal Cutting Temperature Compound embedding.

Cell Culture. The LS174T cell line, a gift from Dr Rex Gaskins (University of Illinois, Champaign, IL) was maintained per the recommendations of American Type Culture Collection (Manassas, VA), and plated on six-well culture plates for assays. For secretion assays, cells were serum-starved for $1 \mathrm{~h}$, then fresh media was added along with $10 \mu \mathrm{M} \mathrm{CCh}$, and incubated for $30 \mathrm{~min}$ at $37^{\circ} \mathrm{C}$. The supernatent was centrifuged and Muc5ac was measured using an enzyme-linked immunosorbent assay. In some experiments $10 \mu \mathrm{g} / \mathrm{ml}$ LPS, $10 \mu \mathrm{g} / \mathrm{ml}$ recombinant human EGF (Shenandoah Biotechnologies), $5 \mu \mathrm{l}$ heat-killed CCs, $1 \mu \mathrm{g} / \mathrm{ml}$ tryphostin AG1478 (EGFRi), or $10 \mu \mathrm{g} / \mathrm{ml} \mathrm{U}-0126$ (MAPKi) were added for $30 \mathrm{~min}$ before carbamycholine addition, during serum starvation.

Enzyme-linked immunosorbent assays. Muc5ac secreted by LS174T cells was measured using an enzyme-linked immunosorbent assay comprised of a polyclonal Muc5AC antibody (\#Ab78660, Abcam, Cambridge, UK) for capture, and biotin-labeled monoclonal (\#MS-145-B, Thermo Fisher Scientific, Runcorn, UK) for detection, and compared with a standard curve with recombinant protein (\#H00004586-Q01, Novus Biologicals, Littleton, CO). Phosphorylated p42/p44 MAPK (R\&D Systems, Minneapolis, MN), phosphorylated EGFR (R\&D Systems), IL-6 (R\&D Systems), IL-17 (R\&D Systems), and CXCL1 (R\&D Systems) were measured using an enzyme-linked immunosorbent assay as per the manufacturers' recommendations. Phosphorylated p42/p44 MAPK and phosphorylated EGFR were measured on $1 / 32$ and $1 / 216$, respectively, of the epithelial cell population removed from the intestine or colon by one incubation in HBSS containing $5 \mathrm{~mm}$ EDTA at $37^{\circ} \mathrm{C}$ for $15 \mathrm{~min}$. IL- 6 and IL- 17 were measured from the supernatant of mesenteric lymph node cells and LP cells isolated as described above, and cultured at a density of $5 \times 10^{6} / \mathrm{ml}$ in RPMI media containing $10 \%$ fetal calf serum, $5 \times 10^{-5} \mathrm{M} 2-\mathrm{ME}$, $2 \mathrm{~mm}$ L-glutamine, $10 \mathrm{~mm}$ HEPES, $50 \mathrm{U} / \mathrm{ml}$ penicillin, $50 \mu \mathrm{g} / \mathrm{ml}$ streptomycin, and $1 \mathrm{~mm}$ sodium pyruvate at $37^{\circ} \mathrm{C} 5 \% \mathrm{CO}_{2}$ for 3 days. CXCL1 was measured in whole intestine homogenized in sterile PBS.

Evaluation of gene expression in GCs. RNA was extracted from flow cytometrically sorted GC populations identified and isolated as described above. Gene expression microarrays were performed on four independent isolates of SI or colon GCs by the Genome Technology Access Center at the Washington University School of Medicine using the Illumina Mouse 8 TotalPrep platform (Illumina, San Diego, CA). Analysis of microarray data was performed using Partek Genomics Suite (St Louis, MO). The microarray data discussed in this publication has been deposited in NCBI's Gene Expression Omnibus and are accessible through GEO Series accession number GSE52418. For quantitative real-time PCR assays, RNA was extracted from GC populations isolated as above, treated with DNAse, and transcribed into complementary DNA using Superscript II reverse transcriptase 
(Invitrogen) according to the manufacturer's recommendations. Primers used for reverse transcriptase-PCR are described in Supplementary Table S2. The absolute copy number of the target was calculated from standards that were constructed as previously described. ${ }^{49}$

Analysis of luminal antigen delivery to LP-DCs. Mice were anesthetized and $2 \mathrm{mg}$ of ovalbumin (Sigma-Aldrich) dissolved in PBS, or PBS alone (controls) was injected into the lumen of the SI or colon. Two hours after the administration of ovalbumin, cell populations were isolated from the non-follicle-bearing LP as previously described $^{49}$ and sorted by flow cytometry. Sorted DC populations were cultured with flow cytometric-sorted $\mathrm{CD} 3+, \mathrm{CD} 8 \alpha+, \mathrm{V} \alpha 2+$, $\mathrm{V} \beta 8+$ splenic T cells from OTI transgenic mice at a ratio of 1:10 DC to T cells. As a positive control, $20 \mu \mathrm{g}$ of Ova was added to cultures of DC populations isolated from mice receiving luminal PBS. After 3 days, cultures were evaluated for the number of T cells by flow cytometry and cell counting.

Statistical analysis. Data analysis using a Student's $t$-test was performed using GraphPad Prism (GraphPad Software, San Diego, CA). Microarray analysis was preformed with JMP Genomics (SAS Institute, Cary, NC). Statistical significance was defined as $P<0.05$.

SUPPLEMENTARY MATERIAL is linked to the online version of the paper at http://www.nature.com/mi

\section{ACKNOWLEDGMENTS}

Supported by grants: DK064798-RDN, Al095550-RDN, DK097317-RDN, and DK097893-KAK. We thank Dr Mark J. Miller and Dr Marco Colonna for insightful critiques and assistance with $2 \mathrm{P}$ imaging and analysis (M.J.M.). The $2 \mathrm{P}$ imaging was performed at the In Vivo Imaging Core at the Washington University School of Medicine. The Washington University Digestive Diseases Research Center Core (DDRCC) Gnotobiotic Facility, supported by NIH grant P30 DK052574, provided the germ-free mice. The High Speed Cell Sorter Core at the Alvin J. Siteman Cancer Center at Washington University School of Medicine and Barnes-Jewish Hospital in St Louis, MO provided flow cytometric cell sorting services. The Siteman Cancer Center is supported in part by $\mathrm{NCl}$ Cancer Center Support Grant P30 CA91842. The Speed Congenics Facility of the Rheumatic Diseases Core Center, supported by NIH grant P30AR048335, bred the Myd88 ${ }^{-1-}$ mice onto the C57BL/6 background.

\section{AUTHOR CONTRIBUTIONS}

K.A.K., K.G.M., and J.R.M. performed two-photon imaging experiments and data analysis. K.A.K. performed cell isolation, ex vivo and in vitro studies, immunofluorescene, ELISA, microarray analysis, flow cytometry, and data analysis. K.A.K. and S.M. performed RT-PCR and data analysis. K.A.K. and R.D.N. conceived the study, directed the experiment design, analyzed the data, and wrote the manuscript. All authors reviewed and discussed the manuscript.

\section{DISCLOSURE}

The authors declare no conflict of interest.

(c) 2015 Society for Mucosal Immunology

\section{REFERENCES}

1. Eri, R. \& Chieppa, M. Messages from the inside. The dynamic environment that favors intestinal homeostasis. Front. Immunol. 4, 323 (2013).

2. Peterson, L.W. \& Artis, D. Intestinal epithelial cells: regulators of barrier function and immune homeostasis. Nat. Rev. Immunol. 14, 141-153 (2014).

3. Johansson, M.E. \& Hansson, G.C. Is the intestinal goblet cell a major immune cell?. Cell Host Microbe 15, 251-252 (2014).

4. Kim, Y.S. \& Ho, S.B. Intestinal goblet cells and mucins in health and disease: recent insights and progress. Curr. Gastroenterol. Rep. 12, 319-330 (2010).
5. Taupin, D. \& Podolsky, D.K. Trefoil factors: initiators of mucosal healing. Nat. Rev. Mol. Cell Biol. 4, 721-732 (2003).

6. Herbert, D.R. et al. Intestinal epithelial cell secretion of RELM-beta protects against gastrointestinal worm infection. J. Exp. Med. 206, 2947-2957 (2009).

7. Johansson, M.E., Thomsson, K.A. \& Hansson, G.C. Proteomic analyses of the two mucus layers of the colon barrier reveal that their main component, the Muc2 mucin, is strongly bound to the Fcgbp protein. J. Proteome. Res. 8, 3549-3557 (2009).

8. McDole, J.R. et al. Goblet cells deliver luminal antigen to CD103+ dendritic cells in the small intestine. Nature 483, 345-349 (2012).

9. Epple, H.J. et al. Differential stimulation of intestinal mucin secretion by cholera toxin and carbachol. Pflugers Arch. 433, 638-647 (1997).

10. Hodges, R.R. et al. Signaling pathways used by EGF to stimulate conjunctival goblet cell secretion. Exp. Eye Res. 103, 99-113 (2012).

11. Wess, J., Eglen, R.M. \& Gautam, D. Muscarinic acetylcholine receptors: mutant mice provide new insights for drug development. Nat. Rev. Drug Discov. 6, 721-733 (2007).

12. Neish, A.S. Microbes in gastrointestinal health and disease. Gastroenterology 136, 65-80 (2009).

13. Shroyer, N. et al. Intestine-specific ablation of mouse atonal homolog 1 (Math1) reveals a role in cellular homeostasis. Gastroenterology 132 , 2478-2488 (2007).

14. Shroyer, N.F., Wallis, D., Venken, K.J., Bellen, H.J. \& Zoghbi, H.Y. Gfi1 functions downstream of Math1 to control intestinal secretory cell subtype allocation and differentiation. Genes Dev. 19, 2412-2417 (2005).

15. Koff, J.L., Shao, M.X., Ueki, I.F. \& Nadel, J.A. Multiple TLRs activate EGFR via a signaling cascade to produce innate immune responses in airway epithelium. Am. J. Physiol. Lung Cell. Mol. Physiol. 294, L1068-L1075 (2008).

16. Cario, E. et al. Lipopolysaccharide activates distinct signaling pathways in intestinal epithelial cell lines expressing toll-like receptors. J. Immunol. 164, 966-972 (2000).

17. Keely, S.J., Calandrella, S.O. \& Barrett, K.E. Carbachol-stimulated transactivation of epidermal growth factor receptor and mitogen-activated protein kinase in T(84) cells is mediated by intracellular ca(2+), PYK-2, and p60(src). J. Biol. Chem. 275, 12619-12625 (2000).

18. Dartt, D.A. Neural regulation of lacrimal gland secretory processes: relevance in dry eye diseases. Prog. Retin. Eye Res. 28, 155-177 (2009).

19. Ota, I. et al. Alpha 1-adrenergic and cholinergic agonists activate MAPK by separate mechanisms to inhibit secretion in lacrimal gland. Am. J. Physiol. Cell. Physiol. 284, C168-C178 (2003).

20. Lindquist, R.L. et al. Visualizing dendritic cell networks in vivo. Nat. Immunol. 5, 1243-1250 (2004).

21. Knoop, K.A., Miller, M.J. \& Newberry, R.D. Transepithelial antigen delivery in the small intestine: different paths, different outcomes. Curr. Opin. Gastroenterol. 29, 112-118 (2013).

22. Spahn, T.W. \& Kucharzik, T. Modulating the intestinal immune system: the role of lymphotoxin and GALT organs. Gut 53, 456-465 (2004).

23. Worbs, T. et al. Oral tolerance originates in the intestinal immune system and relies on antigen carriage by dendritic cells. J. Exp. Med. 203, 519-527 (2006).

24. Shen, L., Weber, C.R., Raleigh, D.R., Yu, D. \& Turner, J.R. Tight junction pore and leak pathways: a dynamic duo. Annu. Rev. Physiol. 73, 283-309 (2011).

25. Turner, J.R. Intestinal mucosal barrier function in health and disease. Nat. Rev. Immunol. 9, 799-809 (2009).

26. Su, L. et al. Targeted epithelial tight junction dysfunction causes immune activation and contributes to development of experimental colitis. Gastroenterology 136, 551-563 (2009).

27. Jang, M.H. et al. Intestinal villous $M$ cells: an antigen entry site in the mucosal epithelium. Proc. Natl. Acad. Sci. USA 101, 6110-6115 (2004).

28. Knoop, K.A. et al. RANKL is necessary and sufficient to initiate development of antigen-sampling $\mathrm{M}$ cells in the intestinal epithelium. J. Immunol. 183, 5738-5747 (2009).

29. Terahara, K. et al. Comprehensive gene expression profiling of Peyer's patch M cells, villous M-like cells, and intestinal epithelial cells. J. Immunol. 180, 7840-7846 (2008). 
30. Rescigno, M. et al. Dendritic cells express tight junction proteins and penetrate gut epithelial monolayers to sample bacteria. Nat. Immunol. 2, 361-367 (2001).

31. Niess, J.H. CX3CR1-mediated dendritic cell access to the intestinal lumen and bacterial clearance. Science 307, 254-258 (2005).

32. Chieppa, M., Rescigno, M., Huang, A.Y. \& Germain, R.N. Dynamic imaging of dendritic cell extension into the small bowel lumen in response to epithelial cell TLR engagement. J. Exp. Med. 203, 2841-2852 (2006).

33. Vallon-Eberhard, A., Landsman, L., Yogev, N., Verrier, B. \& Jung, S. Transepithelial pathogen uptake into the small intestinal lamina propria. J. Immunol. 176, 2465-2469 (2006).

34. Farache, J. et al. Luminal bacteria recruit CD103 + dendritic cells into the intestinal epithelium to sample bacterial antigens for presentation. Immunity 38, 581-595 (2013).

35. Shan, M. et al. Mucus enhances gut homeostasis and oral tolerance by delivering immunoregulatory signals. Science 342, 447-453 (2013).

36. Specian, R.D. \& Neutra, M.R. Mechanism of rapid mucus secretion in goblet cells stimulated by acetylcholine. J. Cell Biol. 85, 626-640 (1980).

37. Neutra, M.R., O'Malley, L.J. \& Specian, R.D. Regulation of intestinal goblet cell secretion. II. A survey of potential secretagogues. Am. J. Physiol. 242, G380-G387 (1982).

38. Pickett, J.A. \& Edwardson, J.M. Compound exocytosis: mechanisms and functional significance. Traffic 7, 109-116 (2006).

39. Mazzini, E., Massimiliano, L., Penna, G. \& Rescigno, M. Oral tolerance can be established via gap junction transfer of fed antigens from CX3CR1 macrophages to CD103 dendritic cells. Immunity 40, 248-261 (2014).

40. Johansson, M.E., Larsson, J.M. \& Hansson, G.C. The two mucus layers of colon are organized by the MUC2 mucin, whereas the outer layer is a legislator of host-microbial interactions. Proc. Natl. Acad. Sci. USA 108 (Suppl 1), 4659-4665 (2011).

41. Ermund, A., Gustafsson, J.K., Hansson, G.C. \& Keita, A.V. Mucus properties and goblet cell quantification in mouse, rat and human ileal Peyer's patches. PLoS One 8, e83688 (2013).

42. Hogquist, K.A. et al. T-cell receptor antagonist peptides induce positive selection. Cell 76, 17-27 (1994).

43. Rose, M.F., Ahmad, K.A., Thaller, C. \& Zoghbi, H.Y. Excitatory neurons of the proprioceptive, interoceptive, and arousal hindbrain networks share a developmental requirement for Math1. Proc. Natl. Acad. Sci. USA 106, 22462-22467 (2009).

44. Jung, S. et al. Analysis of fractalkine receptor CX(3)CR1 function by targeted deletion and green fluorescent protein reporter gene insertion. Mol. Cell. Biol. 20, 4106-4114 (2000).

45. Madisen, L. et al. A robust and high-throughput Cre reporting and characterization system for the whole mouse brain. Nat. Neurosci. 13, 133-140 (2010).

46. Adachi, O. et al. Targeted disruption of the MyD88 gene results in loss of IL-1- and IL-18-mediated function. Immunity 9, 143-150 (1998).

47. el Marjou, F. et al. Tissue-specific and inducible Cre-mediated recombination in the gut epithelium. Genesis 39, 186-193 (2004).

48. McDonald, K.G. et al. Epithelial expression of the cytosolic retinoid chaperone cellular retinol binding protein II is essential for in vivo imprinting of local gut dendritic cells by lumenal retinoids. Am. J. Pathol. 180, 984-997 (2012).

49. McDonald, K.G., McDonough, J.S., Dieckgraefe, B.K. \& Newberry, R.D. Dendritic cells produce CXCL13 and participate in the development of murine small intestine lymphoid tissues. Am. J. Pathol. 176, 2367-2377 (2010). 